\title{
Robust event-triggered output feedback controllers for nonlinear systems
}

\author{
Mahmoud Abdelrahim ${ }^{a}$, Romain Postoyan $^{b, c}$, Jamal Daafouz $^{b, c}$, Dragan Nešić $^{d}$ \\ a Department of Mechanical Engineering, Eindhoven University of Technology, The Netherlands \\ b Université de Lorraine, CRAN, UMR 7039, France \\ c CNRS, CRAN, UMR 7039, France \\ d Department of Electrical and Electronic Engineering, the University of Melbourne, Australia
}

\begin{abstract}
We address the robust stabilization of nonlinear systems subject to exogenous inputs using event-triggered output feedback laws. The plant dynamics is affected by external disturbances, while the output measurement and the control input are corrupted by noises. The communication between the plant and the controller is ensured by a digital channel. The feedback law is constructed in continuous-time, meaning that we ignore the communication network at this step. We then design the sampling rule to preserve stability. Two implementation scenarios are investigated. We first consider the case where the sampling of the plant measurements and of the control input are generated by the same rule, which leads to synchronous transmissions. We then study the scenario where two different laws are used to sample the measurements on the one hand, and the control input on the other hand, thus leading to asynchronous transmissions. In both cases, the transmission conditions consist in waiting a fixed amount of time after each sampling instant and then in checking a state-dependent criterion: when the latter is violated, a transmission occurs. In that way, Zeno phenomenon is a fortiori excluded. The proposed hybrid controllers are shown to ensure either an input-to-state stability property or an $\mathcal{L}_{p}$ stability property, depending on the assumptions. The results are applied to linear time-invariant systems as a particular case, for which the assumptions are formulated as linear matrix inequalities. The proposed strategy encompasses time-driven (and so periodic) sampling as a particular case, for which the results are new. The effectiveness of the approach is illustrated on simulations for a physical system.
\end{abstract}

\section{Introduction}

A recent trend in technology is to connect the control system with the plant via a digital communication channel. This configuration is referred to as networked control systems (NCS) and has many advantages over point-topoint connections in terms of reduced cost, flexibility, and ease of maintenance, see e.g. [9] and the references therein. As the network has a limited bandwidth, one of the challenges is to design control solutions which do not excessively use the communication channel $[13,23]$.

\footnotetext{
ऋ This work was partially supported by the ANR under the grant COMPACS (ANR-13-BS03-0004-02). M. Abdelrahim work is supported by the Dutch Science Foundation (STW) and the Dutch Organization for Scientific Research (NWO) under the VICI grant "Wireless control systems: A new frontier in automation". D. Nešić work is supported by the Australian Research Council under the Discovery Projects scheme.
}

Email addresses: m.m.o.abdelrahim@tue.nl (Mahmoud Abdelrahim), romain.postoyan@univ-lorraine.fr (Romain Postoyan), jamal.daafouz@univ-lorraine.fr (Jamal Daafouz), dnesic@unimelb.edu.au (Dragan Nešić).
To that end, many researchers have proposed to adapt transmissions to the current state of the plant so that the network is only used when it is necessary in view of the control objectives. In this context, event-triggered and self-triggered strategies have been developed in the literature, see e.g. [22] and the references therein. In eventtriggered control, the next transmission instant is generated based on the last transmitted value and the current value of the plant measurement, e.g. [11, 12,38, 41]. In self-triggered controllers, the sequence of transmission instants only depends on the last transmitted value of the plant measurement, e.g. $[8,31,44,45]$. In this paper, we focus on event-triggered control.

While various event-triggered control techniques have been proposed these last years (see $[22,38]$ and the references therein), robustness remains a largely open question. It has been shown in $[1,14]$ that standard techniques, such as the one in [41], may not be robust, in the sense that Zeno phenomenon may occur, which means an infinite number of sampling instants in finite (ordinary) time. Although the presence of exogenous inputs is inevitable in practice, few adapted event-triggered control solutions are available in the literature, see $[14,18,27,28$, 
30,37]. Among these works, only [14,28] have considered the presence of both plant disturbances and measurement noise, to the best of our knowledge. The results of $[14,28]$ focus on the case where only the plant state or the plant output is transmitted over the network but not the control input. Moreover, the triggering mechanisms in $[14,28]$ are developed for static controllers. Note that the techniques of [14] (for the output feedback case) and [28] are dedicated to linear time-invariant (LTI) systems. We have recently become aware of the work of [17] where the authors studied the $\mathcal{L}_{p}$ stabilization of nonlinear systems by using output-based dynamic event-triggered controllers. In comparison to this recent result, we consider different types of exogenous inputs, the proposed triggering condition is different from the one developed in [17], we allow the asynchronous transmissions of the output measurement and of the control input, and we ensure different stability properties.

In this paper, we consider the scenario where the plant dynamics is nonlinear, and depends on external disturbances. Moreover, the output measurements and the control inputs are broadcast over the network and are possibly affected by noise. We first study the case when the output measurements and the control input are synchronously transmitted over the network using a single event-triggering condition. This set-up covers the situations where only the output measurement (equivalently the control input) is sampled and the controller is colocated with the actuators (equivalently with the sensors) as a particular case. We also provide a solution when the (noisy) plant measurements and the (noisy) controller output are sampled according to two distinct rules: we talk of asynchronous transmissions. This setup is relevant when the controller is dynamic and is not co-located with the sensors and the actuators. In both scenarios, the triggering condition only depends on locally available information, i.e. the output measurement and/or the control input, which are corrupted by noises, as well as the clocks designed by the user. Our objective is to design event-triggered controllers that robustly stabilize the system, in a sense we make precise below.

We follow the emulation approach to synthesize the event-triggered controllers. We first assume that the plant can be stabilized in the absence of network by an output feedback law. Then, we take into account the effect of network and we synthesize appropriate eventtriggering conditions such that the closed-loop stability is preserved. The event-triggering mechanism combines techniques from time-triggered control, inspired by [35], and event-triggered implementation [41]. The idea is to wait $T$ units of time after each transmission, where $T>0$ is based on the maximally allowable transmission interval (MATI) given by periodic sampling, and to evaluate, afterwards, an output-dependent and/or input-dependent criterion. In that way, a strictly positive lower bound on the inter-transmission times is enforced, which rules out Zeno solutions. For this pur- pose, we had to develop new MATI bounds compared to [35] to handle the effect of exogenous inputs as well as asynchronous sampling. We provide sufficient conditions under which robustness of the closed-loop system is guaranteed against exogenous inputs. In particular, we ensure an input-to-state stability (ISS) or an $\mathcal{L}_{p}$ $\left(\mathrm{D} \mathcal{L}_{p}\right)$ stability in the sense of [7] depending on the assumptions. The idea of enforcing a lower bound on the inter-transmission times is related to time regularization techniques, see [25], and has been applied in e.g. $[17,19,32,42,46]$. What distinguishes our work from these references (with the exception of [17]) is that the minimum time that we enforce between two consecutive transmission instants is designed based on time-triggered control results. Furthermore, our technique applies to both linear and nonlinear systems and we consider more general implementation/perturbation scenarios than those considered in $[17,19,32,42,46]$.

Our approach applies to any stabilizable and detectable linear time-invariant (LTI) systems, for which the results are new. In this case, we reformulate the assumptions as a linear matrix inequality (LMI). The results are also new in the particular case of time-triggered control as the minimum time $T$ between two transmissions mentioned above can be used as a maximum sampling period. In this context, we are only aware of the works [10,21] for the asynchronous time-triggered implementations. Note that the proposed schemes in $[10,21]$ are dedicated to the disturbance-free case. Moreover, the implementation scenarios and the set of assumptions that we consider are different from those in $[10,21]$. The effectiveness of the approach is demonstrated on a nonlinear model of a single-link robot arm. The numerical simulations illustrate a tradeoff between the estimated $\mathcal{L}_{p}$ gain and the lower bound on the inter-transmission times that we enforce, as also shown in [17]. This tradeoff is characterized by some design parameters which can be tuned by the user to adapt the hardware constraints and/or the desired performance.

To summarize, the main contributions of the paper are methodologies to design event-triggered controllers, which robustly stabilize a class of nonlinear systems either when the transmissions are synchronous or not. This work generalizes our results in [2] to the case of asynchronous event-triggered control and/or to the case where the plant, the output measurement and the control input are affected by exogenous inputs. Compared to $[5,6]$, the result in $[6]$ deals with the scenario where both the output measurement and the control input are transmitted synchronously, while the technique in [5] has been developed in the absence of exogenous inputs. Moreover, these references focus on input-to-state stability and do not address $\mathcal{L}_{p}$ stabilization.

The rest of the paper is organised as follows. Preliminaries are given in Section 2. The synchronous eventtriggered implementation is studied in Section 3. The 
asynchronous scenario is investigated in Section 4. The application to LTI systems is presented in Section 5. We illustrate the approach on a nonlinear model of a singlelink robot arm in Section 6. Conclusions are provided in Section 7. The proofs are given in the Appendix.

\section{Preliminaries}

Let $\mathbb{R}:=(-\infty, \infty), \mathbb{R}_{>0}:=[0, \infty), \mathbb{Z}_{\geq 0}:=\{0,1,2, \ldots\}$ and $\mathbb{Z}_{>0}:=\{1,2, \ldots\}$. A continuous function $\gamma: \mathbb{R}_{\geq 0} \rightarrow$ $\mathbb{R}_{\geq 0}$ is of class $\mathcal{K}$ if it is zero at zero, strictly increasing, and it is of class $\mathcal{K}_{\infty}$ if in addition $\gamma(s) \rightarrow \infty$ as $s \rightarrow \infty$. A continuous function $\gamma: \mathbb{R}_{>0}^{2} \rightarrow \mathbb{R}_{\geq 0}$ is of class $\mathcal{K} \mathcal{L}$ if for each $t \in \mathbb{R}_{\geq 0}, \gamma(\cdot, t)$ is of class $\mathcal{K}$, and, for each $s \in \mathbb{R}_{\geq 0}$, $\gamma(s, \cdot)$ is decreasing to zero. We denote the minimum and the maximum eigenvalues of the real symmetric matrix $A$ as $\lambda_{\min }(A)$ and $\lambda_{\max }(A)$, respectively. We write $A^{T}$ to denote the transpose of $A$, and $\mathbb{I}_{n}$ stands for the identity matrix of dimension $n$. We write $(x, y)$ to represent the vector $\left[x^{T}, y^{T}\right]^{T}$ for $x \in \mathbb{R}^{n}$ and $y \in \mathbb{R}^{m}$. For a vector $x \in \mathbb{R}^{n}$, we denote by $|x|:=\sqrt{x^{T} x}$ its Euclidean norm and, for a matrix $A \in \mathbb{R}^{n \times m},|A|:=\sqrt{\lambda_{\max }\left(A^{T} A\right)}$.

We consider hybrid systems of the following form $[15,20]$

$$
\dot{x}=F(x, w) \quad x \in \mathcal{C}, \quad x^{+} \in G(x) \quad x \in \mathcal{D},
$$

where $x \in \mathbb{R}^{n_{x}}$ is the state, $w \in \mathbb{R}^{n_{w}}$ is an exogenous input, $\mathcal{C}$ is the flow set, $F$ is the flow map, $\mathcal{D}$ is the jump set and $G$ is the jump map. Note that in (1), the exogenous input $w$ only affects the flow dynamics and the flow and the jump sets do not depend on $w$, which is sufficient for the purpose of this study. We assume that the hybrid model (1) satisfies the basic regularity conditions, see Section 6.2 in [20]. Hence, the vector field $F$ is assumed to be continuous and $G$ to be outer semicontinuous and locally bounded with respect to $\mathcal{D}$, and the sets $\mathcal{C}$ and $\mathcal{D}$ are assumed to be closed, which will be the case in our study.

We recall some definitions from $[15,20]$. Solutions to system (1) are defined on so-called hybrid time domains. A set $E \subset \mathbb{R}_{\geq 0} \times \mathbb{Z}_{\geq 0}$ is called a compact hybrid time domain if $E=\bigcup_{j=0}^{J-1}\left(\left[t_{j}, t_{j+1}\right], j\right)$ for some finite sequence of times $0=t_{0} \leq t_{1} \leq \ldots \leq t_{J}$ and it is a hybrid time domain if for all $(T, \bar{J}) \in \bar{E}, E \cap([0, T] \times\{0,1, \ldots, J\})$ is a compact hybrid time domain. A hybrid signal is a function defined on a hybrid time domain. A hybrid signal $w: \operatorname{dom} w \rightarrow \mathbb{R}^{n_{w}}$ is called a hybrid input if $w(\cdot, j)$ is measurable and locally essentially bounded for each $j$. A hybrid signal $x: \operatorname{dom} x \rightarrow \mathbb{R}^{n_{x}}$ is called a hybrid arc if $x(\cdot, j)$ is locally absolutely continuous for each $j$. A hybrid arc $x: \operatorname{dom} x \rightarrow \mathbb{R}^{n_{x}}$ and a hybrid input $w: \operatorname{dom} w \rightarrow \mathbb{R}^{n_{w}}$ is a solution pair $(x, w)$ to system (1) if $\operatorname{dom} x=\operatorname{dom} w, x(0,0) \in \mathcal{C} \cup \mathcal{D}$, and: (i) for all $j \in \mathbb{Z}_{\geq 0}$, and almost all $t$ such that $(t, j) \in \operatorname{dom} x$, $x(t, j) \in \mathcal{C}$ and $\dot{x}(t, j)=F(x(t, j), w(t, j))$; (ii) for all $(t, j) \in \operatorname{dom} x$ such that $(t, j+1) \in \operatorname{dom} x, x(t, j) \in \mathcal{D}$ and $x(t, j+1) \in G(x(t, j), w(t, j))$.

The following definition of $\mathcal{L}_{\infty}$ norm for hybrid signals was proposed in [15].

Definition 1 ( $\mathcal{L}_{\infty}$ norm [15]) Given a hybrid signalr, its $\mathcal{L}_{\infty}$ norm is given by

$$
\begin{aligned}
& \|r\|_{\infty}:=\max \left\{\begin{array}{l}
\operatorname{ess} \sup _{\left(t^{\prime}, j^{\prime}\right) \in \operatorname{dom} r \backslash \Gamma(r), t^{\prime}+j^{\prime} \leq t+j}\left|r\left(t^{\prime}, j^{\prime}\right)\right|,
\end{array}\right. \\
& \left.\sup _{\left(t^{\prime}, j^{\prime}\right) \in \Gamma(r), t^{\prime}+j^{\prime} \leq t+j}\left|r\left(t^{\prime}, j^{\prime}\right)\right|\right\},
\end{aligned}
$$

where $\Gamma(r):=\{(t, j) \in \operatorname{dom} r:(t, j+1) \in \operatorname{dom} r\}$. If the right-hand side in (2) exists and is finite, we write $w \in \mathcal{L}_{\infty}$.

We adopt the following notions of $\mathcal{L}_{p}$ norm with $p \in \mathbb{Z}_{>0}$ $[23]$.

Definition $2\left(\mathcal{L}_{p}\right.$ norm [23]) For a hybrid signal $z$ defined on the hybrid time domain dom $z=\bigcup_{j=0}^{J-1}\left(\left[t_{j}, t_{j+1}\right], j\right)$ with $J$ possibly $\infty$ and/ort $t_{J}=\infty$, the $\mathcal{L}_{p}$ norm of $z$ is defined as, for $p \in \mathbb{Z}_{>0},\|z\|_{p}:=\left(\sum_{j=0}^{J-1} \int_{t_{j}}^{t_{j+1}}|z(t, j)|^{p} d t\right)^{\frac{1}{p}}$ provided that the right-hand side exists and is finite. In this case, we write $z \in \mathcal{L}_{p}$.

Based on Definition 2, we can define $\mathcal{L}_{p}$ stability for system (1).

Definition $3\left(\mathcal{L}_{p}\right.$ stability [23]) Given $p \in \mathbb{Z}_{>0}$, system (1) is $\mathcal{L}_{p}$ stable from the input $w$ to the output $z:=h(x, w)$ with gain less than or equal to $\eta \geq 0$ if there exists $\beta \in \mathcal{K}_{\infty}$ such that any solution pair $(x, w)$ to (1) satisfies $\|z\|_{p} \leq \beta(|x(0,0)|)+\eta\|w\|_{p}$.

\section{Synchronous event-triggered implementation}

\subsection{Hybrid model and problem statement}

Consider the nonlinear plant model

$$
\dot{x}_{p}=f_{p}\left(x_{p}, u, w\right), \quad y=g_{p}\left(x_{p}, d_{y}\right),
$$

where $x_{p} \in \mathbb{R}^{n_{p}}$ is the plant state, $u \in \mathbb{R}^{n_{u}}$ is the control input, $w \in \mathbb{R}^{n_{w}}$ is a vector of unknown exogenous disturbances, $y \in \mathbb{R}^{n_{y}}$ is the available output of the plant, which is affected by noise $d_{y} \in \mathbb{R}^{n_{y}}$. We assume that the 
signal corresponding to $d_{y}$ is differentiable. We consider the following dynamic controller

$$
\dot{x}_{c}=f_{c}\left(x_{c}, y\right), \quad u=g_{c}\left(x_{c}, y, d_{u}\right),
$$

where $x_{c} \in \mathbb{R}^{n_{c}}$ is the controller state and $d_{u} \in \mathbb{R}^{n_{u}}$ is noise corrupting the control input. The noise $d_{u}$ may model computational glitches or quantization errors or more generally any disturbance, which may affect the control input. We assume that the signal corresponding to $d_{u}$ is differentiable. Moreover, we assume that controller (4) is not necessarily observer-based and it captures static feedback controllers as a particular case when $u=g_{c}\left(y, d_{u}\right)$. Note that both the control input in (4) involves a feedthrough term. The functions $f_{p}, f_{c}$ are assumed to be continuous and the functions $g_{p}, g_{c}$ are assumed to be continuously differentiable.

We consider the scenario where plant (3) and controller (4) communicate with each other through a digital channel. The plant output $y$ and the control input $u$ are sent over the network to the controller and the plant, respectively, at discrete instants $t_{i}, i \in \mathcal{I} \subseteq \mathbb{Z}_{\geq 0}$. The sequence of transmission instants is defined by an event-triggering mechanism. For the sake of generality, we allow the triggering condition to depend on both $y$ and $u$. In that way, we cover the common situations where only the plant output (respectively, the control input) is sent over the network and the controller is co-located with actuators (respectively, the sensors), see Figure 1. In these two cases, the triggering rule respectively depends on $y$ only and on $(y, u)$, in addition to an auxiliary clock variable that we will introduce in the sequel. When both $y$ and $u$ are sent over the network, it may be difficult to implement the strategy proposed in this section in practice. It makes more sense in this case to design distinct triggering policies for $y$ and $u$ : this is the purpose of Section 4 .

At each transmission instant, $y$ is sent to the controller which computes a new control input that is instantaneously transmitted to the plant. We ignore the effect of small transmission and computation delays, which can be handled like in [41].

The overall system is described by the equations below

$$
\begin{aligned}
\dot{x}_{p} & =f_{p}\left(x_{p}, \hat{u}, w\right) & & t \in\left[t_{i}, t_{i+1}\right] \\
\dot{x}_{c} & =f_{c}\left(x_{c}, \hat{y}\right) & & t \in\left[t_{i}, t_{i+1}\right] \\
y & =g_{p}\left(x_{p}, d_{y}\right) & & \\
u & =g_{c}\left(x_{c}, \hat{y}, d_{u}\right) & & \\
\dot{\hat{y}} & =0 & & t \in\left[t_{i}, t_{i+1}\right] \\
\dot{\hat{u}} & =0 & & t \in\left[t_{i}, t_{i+1}\right] \\
\hat{y}\left(t_{i}^{+}\right) & =y\left(t_{i}\right) & & \\
\hat{u}\left(t_{i}^{+}\right) & =u\left(t_{i}\right), & &
\end{aligned}
$$

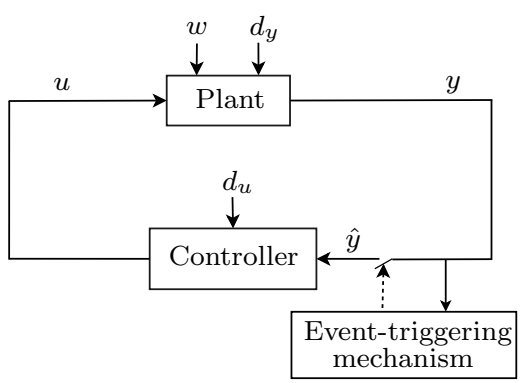

(a)

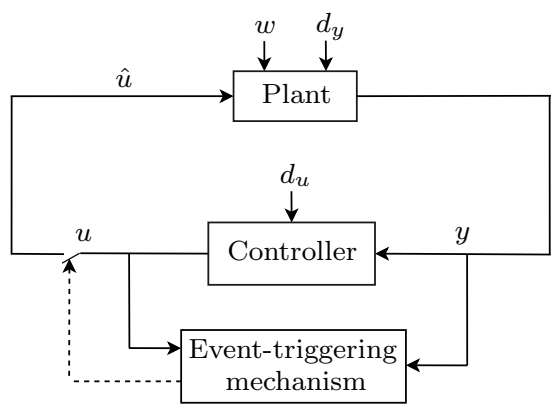

(b)

Fig. 1. Implementation setup for particular cases: (a) only $y$ is transmitted over the network; (b) only $u$ is transmitted over the network.

where $\hat{y}$ and $\hat{u}$ respectively denote the last transmitted values of $y$ and $u$. We assume that zero-order-hold implementations are used to generate the sampled values $\hat{y}$ and $\hat{u}$ on flows, which leads to $\dot{\hat{y}}=0$ and $\dot{\hat{u}}=0$ between two successive sampling instants in (5).

Because we only have access to noisy signals, we define the sampling-induced error using noisy variables. Hence, we consider $e:=\left(e_{y}, e_{u}\right) \in \mathbb{R}^{n_{e}}$, where

$$
\begin{aligned}
e_{y} & :=\hat{y}-y \\
e_{u} & :=\hat{u}-u
\end{aligned}
$$

which are both reset to 0 at each transmission instant. Note that $e$ is available to the event-triggering mechanism. We also introduce an additional clock variable $\tau \in \mathbb{R}$ to describe the time elapsed since the last transmission, which has the following dynamics

$$
\dot{\tau}=1 \quad t \in\left[t_{i}, t_{i+1}\right], \quad \tau\left(t_{i}^{+}\right)=0 .
$$

This variable will be useful to define the triggering con- 


$$
\begin{aligned}
& f(x, e, \xi)=\left(\begin{array}{c}
f_{p}\left(x_{p}, g_{c}\left(x_{c}, y+e_{y}, d_{u}\right)+e_{u}, w\right) \\
f_{c}\left(x_{c}, y+e_{y}\right)
\end{array}\right) \\
& g(x, e, \xi)=\left(\begin{array}{c}
-\frac{\partial}{\partial x_{p}} g_{p}\left(x_{p}, d_{y}\right) f_{p}\left(x_{p}, g_{c}\left(x_{c}, y+e_{y}, d_{u}\right)+e_{u}, w\right)-\frac{\partial}{\partial d_{y}} g_{p}\left(x_{p}, d_{y}\right) \dot{d}_{y} \\
-\frac{\partial}{\partial x_{c}} g_{c}\left(x_{c}, y+e_{y}, d_{u}\right) f_{c}\left(x_{c}, y+e_{y}\right)-\frac{\partial}{\partial d_{u}} g_{c}\left(x_{c}, y+e_{y}, d_{u}\right) \dot{d}_{u}
\end{array}\right)
\end{aligned}
$$

dition. In that way, the system is modeled as

$$
\left(\begin{array}{c}
\dot{x} \\
\dot{e} \\
\dot{\tau}
\end{array}\right)=\left(\begin{array}{c}
f(x, e, \xi) \\
g(x, e, \xi) \\
1
\end{array}\right) q \in \mathcal{C}, \quad\left(\begin{array}{c}
x^{+} \\
e^{+} \\
\tau^{+}
\end{array}\right)=\left(\begin{array}{c}
x \\
0 \\
0
\end{array}\right) q \in \mathcal{D},
$$

where $x:=\left(x_{p}, x_{c}\right) \in \mathbb{R}^{n_{x}}, q:=(x, e, \tau) \in \mathbb{R}^{n_{q}}$, and $\xi:=$ $\left(w, d_{y}, d_{u}, \dot{d}_{y}, \dot{d}_{u}\right) \in \mathbb{R}^{n_{\xi}}$ is the concatenation of all the exogenous inputs to (8) with $n_{\xi}:=n_{w}+2 n_{y}+2 n_{u}$ and $\dot{d}_{y}, \dot{d}_{u}$ are the time-derivative of the noises corresponding to $d_{y}$ and $d_{u}$, respectively. The latter appears in the definition of $g$, i.e. of the dynamics of $e$ on flows. The functions $f$ and $g$ in (8) are given in (9).

Our objective is to synthesize output-based triggering conditions, i.e. to design the flow and the jump sets of system (8) using conditions which only involve $e, y, u$ and $\tau$, to ensure stability properties for system (8), as well as the existence of a uniform strictly positive lower bound on the inter-transmission times.

\subsection{Assumptions}

We make the following assumption on system (8), which is inspired by [35].

Assumption 1 There exist locally Lipschitz functions $V: \mathbb{R}^{n_{x}} \rightarrow \mathbb{R}_{\geq 0}, W: \mathbb{R}^{n_{e}} \rightarrow \mathbb{R}_{\geq 0}$ with $W$ positive definite, a continuous function $H: \mathbb{R}^{n_{x}+n_{\xi}} \rightarrow \mathbb{R}_{\geq 0}$, real numbers $L \geq 0, \gamma>0, \underline{\alpha}, \bar{\alpha} \in \mathcal{K}_{\infty}$, a continuous function $\alpha_{s}: \mathbb{R}^{n_{x}+n_{\xi}} \rightarrow \mathbb{R}$, and a continuous function $\delta: \mathbb{R}^{n_{y}} \rightarrow$ $\mathbb{R}_{\geq 0}$ such that:

(i) for all $x \in \mathbb{R}^{n_{x}}$

$$
\underline{\alpha}(|x|) \leq V(x) \leq \bar{\alpha}(|x|) ;
$$

(ii) for almost all $x \in \mathbb{R}^{n_{x}}$ and all $(e, \xi) \in \mathbb{R}^{n_{e}+n_{\xi}}$

$$
\begin{aligned}
\langle\nabla V(x), f(x, e, \xi)\rangle \leq & -\alpha_{s}(x, \xi)-H^{2}(x, \xi)-\delta(y) \\
& +\gamma^{2} W^{2}(e)
\end{aligned}
$$

(iii) for almost all $e \in \mathbb{R}^{n_{e}}$ and all $(x, \xi) \in \mathbb{R}^{n_{x}+n_{\xi}}$

$$
\langle\nabla W(e), g(x, e, \xi)\rangle \leq L W(e)+H(x, \xi) .
$$

Item (i) of Assumption 1 means that $V$ is positive definite and radially unbounded. Item (ii) of Assumption 1 is a dissipativity property of the system $\dot{x}=f(x, e, \xi)$ with inputs $\xi$ and $e$. We will focus on the case where $\alpha_{s}(x, \xi)=\alpha(|x|)-\varrho(|\xi|)$, where $\alpha, \varrho \in \mathcal{K}_{\infty}$ to guarantee an input-to-state stability for system (8). In this case, (11) implies that the system $\dot{x}=f(x, e, \xi)$ is ISS with respect to $e$ and $w$, and input-to-state stable (ISS) with respect to $d_{y}$ and $d_{u}$ in the sense of [7] (since $\varrho \in \mathcal{K}_{\infty}$ and $W$ is positive definite and continuous, $W(e)$ can therefore be upper-bounded by a class- $\mathcal{K}_{\infty}$ function of $|e|$ in view of Lemma 4.3 in [26]). Conditions (10)-(11) also imply that the system $\dot{x}=f(x, e, \xi)$ is $\mathcal{L}_{2}$ gain stable from $(W, \sqrt{\varrho})$ to $(H, \sqrt{\delta})$. We will also consider the case where $\alpha_{s}(x, \xi)=|z|^{p}-\eta^{p}|\xi|^{p}$, with $p \in \mathbb{Z}_{>0}$ and $\eta \geq 0$, to address $\mathcal{L}_{p}$ stabilization. Condition (12) is an exponential growth condition of the $e$ system on flows, which is also used in $[2,6,17,34,35]$. Assumption 1 can always be satisfied for stabilizable and detectable LTI plants (3), see Section 5. A nonlinear example is provided in Section 6.

\subsection{Event-triggering mechanism}

In view of Assumption 1, we could follow the same idea as in [41] and trigger transmission whenever $\gamma^{2} W^{2}(e) \geq$ $\delta(y)$ to (approximately) preserve the dissipativity property of the continuous-time closed-loop system. However, Zeno phenomenon may occur in this case, first, due to the presence of exogenous inputs as explained in [14], and second, because only an output of the plant is used to synthesize the event-triggering condition and not the full state information, see [18] for more detail. To overcome these issues, we enforce the existence of a uniform strictly positive lower bound on the intertransmission times by augmenting the event-triggering condition $\gamma^{2} W^{2}(e) \geq \delta(y)$ with a time-triggering rule. We rely for this purpose on the results of [35], which we extend to the case where exogenous inputs affect the plant. Hence, we define the event-triggering mechanism as follows

$$
\gamma^{2} W^{2}(e) \geq \delta(y) \text { and } \tau \geq T,
$$

where $\gamma, W, \delta$ come from Assumption 1 and $T>0$ is selected such that $T<\mathcal{T}(\kappa, \gamma, L)$, where

$$
\mathcal{T}(\kappa, \gamma, L):= \begin{cases}\frac{1}{L r} \arctan (r) & (1+\kappa) \gamma>L \\ \frac{1}{L} & (1+\kappa) \gamma=L \\ \frac{1}{L r} \operatorname{arctanh}(r) & (1+\kappa) \gamma<L\end{cases}
$$


with $r:=\sqrt{\left|\left(\frac{(1+\kappa) \gamma}{L}\right)^{2}-1\right|}$ and $L, \gamma$ come from Assumption 1 . The constant $\kappa>0$ is a tuning parameter which can be arbitrarily chosen by the user. Note that $\mathcal{T}(\kappa, \gamma, L)$ is a decreasing function in $\kappa$ and when $\kappa \rightarrow 0$ we recover the MATI bound given by [35] and used in $[2,4]$. We will see that a small value of $\kappa$ yields a large ISS gain estimate, thus suggesting a trade-off between the number of transmissions and performance.

The flow and the jump sets of system (8) are defined as

$$
\begin{aligned}
& \mathcal{C}=\left\{(x, e, \tau): \gamma^{2} W^{2}(e) \leq \delta(y) \text { or } \tau \in[0, T]\right\} \\
& \mathcal{D}=\left\{(x, e, \tau): \gamma^{2} W^{2}(e) \geq \delta(y) \text { and } \tau \geq T\right\}
\end{aligned}
$$

We are ready to state the main results of this section.

\section{4 input-to-state stability}

The theorem below ensures ISS property for system (8) when Assumption 1 holds with $\alpha_{s}(x, \xi)=\alpha(|x|)-\varrho(|\xi|)$, where $\alpha, \varrho \in \mathcal{K}_{\infty}$, and when $H$ can be written as $H(x, \xi)=H(x)+\sigma(|\xi|)$ with $\sigma \in \mathcal{K}_{\infty}$.

Theorem 1 Consider system (8) with the flow and the jump sets defined in (15), where the constant $T$ is such that $T \in(0, \mathcal{T}(\kappa, \gamma, L))$ with $\kappa>0$. Suppose that Assumption 1 holds with $\alpha_{s}(x, \xi)=\alpha(|x|)-\varrho(|\xi|)$ and $H(x, \xi)=H(x)+\sigma(|\xi|)$ for all $(x, \xi) \in \mathbb{R}^{n_{x}+n_{\xi}}$, where $\alpha, \varrho, \sigma \in \mathcal{K}_{\infty}$. There exists $\beta \in \mathcal{K} \mathcal{L}$ such that any solution pair $(\phi, \xi)$, with $\phi:=\left(\phi_{x}, \phi_{e}, \phi_{\tau}\right)$ and $\xi \in \mathcal{L}_{\infty}$, satisfies, for all $(t, j) \in \operatorname{dom} \phi$,

$$
\begin{array}{r}
\left|\phi_{x}(t, j)\right| \leq \max \left\{\beta\left(\left|\left(\phi_{x}(0,0), \phi_{e}(0,0)\right)\right|, t+j\right),\right. \\
\left.\psi\left(\|\xi\|_{\infty}\right)\right\},
\end{array}
$$

where $\psi(s):=\underline{\alpha}^{-1}\left(\rho^{-1}\left(\frac{1}{\varepsilon}\left(\varrho(s)+\frac{1}{\kappa} \sigma^{2}(s)\right)\right)\right)$ for $s \geq 0$, $\rho(s):=\min \left\{\rho_{1}\left(\frac{s}{2}\right), \kappa \gamma \theta \frac{s}{2}\right\}$ for $s \geq 0, \rho_{1} \in \mathcal{K}_{\infty}$, and $\theta, \varepsilon \in(0,1)$. Furthermore, the inter-transmission times are lower bounded by the constant $T$.

Theorem 1 shows that system (8), (15) satisfies an ISS property, see [7], with respect to the external disturbance $w$ and the measurement noise $d$. It is worth mentioning that the argument of the ISS gain $\psi$ in (16) includes the derivative of the measurement noises, $\dot{d}_{y}, \dot{d}_{u}$, because of sampling, see (3), (4) and the definitions of the samplinginduced errors. Indeed, in this case the dynamics of the sampling-induced errors between two transmission instants also depend on $\dot{d}_{y}, \dot{d}_{u}$. This type of results is common in the sampled-data control literature, see [33]. The constants $\theta, \varepsilon$ can be arbitrarily chosen in $(0,1)$. We notice that the tuning parameter $\kappa$ may provide a tradeoff between the guaranteed minimum time between transmissions and the upper bound on the estimated ISS gain $\psi$ in view of $(14),(16)$. The value of $\mathcal{T}(\kappa, \gamma, L)$ can be increased by taking $\kappa$ small, however the upper bound on the ISS (nonlinear) gain $\psi$ in (16) will increase, and vice versa.

Remark 1 The optimization of the estimated ISS gain $\psi$ and guaranteed minimum time $T$ between two transmissions is relevant in practice. However, this task is far from trivial since $\psi$ is a highly nonlinear function and depends on several parameters. The investigation of this point is beyond the scope of this study and may be considered in future research.

\section{$3.5 \mathcal{L}_{p}$ stability}

Consider now the case where Assumption 1 holds with $\alpha_{s}(x, \xi)=|z|^{p}-\eta^{p}|\xi|^{p}$, where $z:=h(x, \xi)$ is the controlled output (which is a priori not measured) and with $h$ is a mapping from $\mathbb{R}^{n_{x}+n_{\xi}}$ to $\mathbb{R}^{n_{z}}, p \in \mathbb{Z}_{>0}$, and $\eta \geq 0$. The following result ensures an $\mathcal{L}_{p}$ stability property of system (8), (15) from the input $\xi$ to $z$.

Theorem 2 Consider system (8) with the flow and the jump sets in (15) and the output $z:=h(x, \xi)$, where the constant $T$ is such that $T \in(0, \mathcal{T}(0, \gamma, L))$. Suppose that Assumption 1 holds with $\alpha_{s}(x, \xi)=|z|^{p}-\eta^{p}|\xi|^{p}$, where $p \in \mathbb{Z}_{>0}$ and $\eta \geq 0$. Then system (8) is $\mathcal{L}_{p}$ stable from $\xi$ to $z$ with an $\mathcal{L}_{p}$ gain less that or equal to $\eta$.

The proof of Theorem 2 follows similar lines as the proof of Theorem 3 given in the next section, it is therefore omitted.

\section{Asynchronous event-triggered implementa- tion}

When controller (4) is dynamic and both $y$ and $u$ are transmitted over the network, the strategy proposed in Section 3 may be difficult to implement as already mentioned. In this section, we design independent eventtriggering conditions for $y$ and $u$, which leads to asynchronous event-triggered control, see Figure 2.

\subsection{Hybrid model}

We respectively denote the sequences of transmissions of $y$ and of $u$ by $t_{i}^{y}, i \in \mathcal{I}_{y} \subseteq \mathbb{Z}_{\geq 0}$, and $t_{i}^{u}, i \in \mathcal{I}_{u} \subseteq \mathbb{Z}_{\geq 0}$. At each transmission instant $t_{i}^{y}$, the current output measurement $y$ is sent to the controller, and at each transmission instant $t_{i}^{u}$, the control input $u$ is broadcasted to the actuators. These sequences of transmissions are defined by two independent triggering conditions, which we design in the following. 


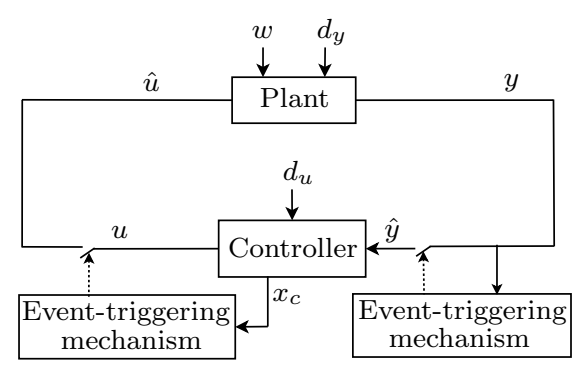

Fig. 2. Asynchronous event-triggered control.

To model the overall system like in Section 3, we need to introduce the sampling-induced errors. We define $e_{y}=\hat{y}-y$ as before but we no longer use $e_{u}=\hat{u}-u$. Indeed, because of the feedthrough term in (4), when $\hat{y}$ is updated, $u$ experiences a jump and so does $e_{u}$. Hence, an update of $\hat{y}$ generates a jump of $e_{u}$, which does not necessarily correspond to an update on the control input (since $\hat{u}$ and $\hat{y}$ are in general not updated at the same time instants). To be more precise, let $t_{i}^{y}, i \in \mathcal{I}_{y} \backslash \mathcal{I}_{u}$. We have $e_{u}\left(t_{i}^{y^{+}}\right)=\hat{u}\left(t_{i}^{y^{+}}\right)-$ $u\left(t_{i}^{y^{+}}\right)=\hat{u}\left(t_{i}^{y}\right)-g_{c}\left(x_{c}\left(t_{i}^{y^{+}}\right), \hat{y}\left(t_{i}^{y^{+}}\right), d_{u}\left(t_{i}^{y^{+}}\right)\right)=\hat{u}\left(t_{i}^{y}\right)-$ $g_{c}\left(x_{c}\left(t_{i}^{y}\right), y\left(t_{i}^{y}\right), d_{u}\left(t_{i}^{y}\right)\right) \neq \hat{u}\left(t_{i}^{y}\right)-g_{c}\left(x_{c}\left(t_{i}^{y}\right), \hat{y}\left(t_{i}^{y}\right), d_{u}\left(t_{i}^{y}\right)\right)$ in general. While it may be possible to handle these jumps of $e_{u}$ in view of $[24,36]$, we prefer to work with $e_{c}:=\hat{x}_{c}-x_{c}$ instead in this section to overcome this issue $^{1}$, where $\hat{x}_{c}$ denotes the sampled-version of $x_{c}$. Note that since $x_{c}$ is the controller state, it is reasonable to assume that this signal is available to the event-triggering mechanism. The value of $\hat{x}_{c}$ is kept constant between two successive sampling instants of the control input and is reset to the actual value of $x_{c}$ when the triggering condition for the input is violated. At each sampling instant $t_{i}^{u}, \hat{x}_{c}$ is updated to the current value of $x_{c}$. As a result, the sampling-induced $e_{c}$ only experiences a jump when $\hat{x}_{c}$ is updated to the current value of $x_{c}$ at $t_{i}^{u}$, which avoids the above mentioned issue. Hence, we consider $e:=\left(e_{y}, e_{c}\right) \in \mathbb{R}^{n_{e}}$ with $n_{e}=n_{y}+n_{c}$. Note that the dimension of the vector of sampling errors $e$ is different from the one considered in Section 3.1. In that

\footnotetext{
1 If the dynamic controller (4) does not involve a directfeedthrough term, i.e. the control input takes the form $u=$ $g_{c}\left(x_{c}, d_{u}\right)$, then we do not have this issue and $e_{u}$ can be defined based on the control input $u$ as in $(6)$, see e.g. $[5,18]$.
}

way, we obtain the impulsive model below

$$
\begin{aligned}
\dot{x}_{p} & =f_{p}\left(x_{p}, \hat{u}, w\right) & & t \in\left[t_{i}^{u}, t_{i+1}^{u}\right] \\
\dot{x}_{c} & =f_{c}\left(x_{c}, \hat{y}\right) & & t \in\left[t_{i}^{y}, t_{i+1}^{y}\right] \\
y & =g_{p}\left(x_{p}, d_{y}\right) & & \\
u & =g_{c}\left(\hat{x}_{c}, \hat{y}, d_{u}\right) & & \\
\dot{\hat{y}} & =0 & & t \in\left[t_{i}^{y}, t_{i+1}^{y}\right] \\
\dot{\hat{x}}_{c} & =0 & & t \in\left[t_{i}^{u}, t_{i+1}^{u}\right] \\
\dot{\hat{u}} & =0 & & t \in\left[t_{i}^{u}, t_{i+1}^{u}\right] \\
\hat{y}\left(t_{i}^{y+}\right) & =y\left(t_{i}^{y}\right) & & \\
\hat{x}_{c}\left(t_{i}^{u+}\right) & =x_{c}\left(t_{i}^{u}\right) & & \\
\hat{u}\left(t_{i}^{u+}\right) & =u\left(t_{i}^{u}\right), & &
\end{aligned}
$$

where $\hat{y}, \hat{x}_{c}$ respectively denote the last transmitted values of the plant output and of the controller state, which are generated by zero-order-holds between two successive transmission instants. In agreement with Section 3, we introduce two timers $\tau_{y}, \tau_{u} \in \mathbb{R}_{>0}$ to describe the time elapsed since the last transmissions of $y$ and of $u$, respectively, which have the following dynamics

$$
\begin{array}{lll}
\dot{\tau}_{y}=1 & t \in\left[t_{i}^{y}, t_{i+1}^{y}\right], & \tau_{y}\left(t_{i}^{y+}\right)=0 \\
\dot{\tau}_{u}=1 & t \in\left[t_{i}^{u}, t_{i+1}^{u}\right], & \tau_{u}\left(t_{i}^{u+}\right)=0 .
\end{array}
$$

Let $q:=\left(x, e_{y}, e_{c}, \hat{u}, \tau_{y}, \tau_{u}\right) \in \mathbb{R}^{n_{q}}, n_{q}:=n_{x}+n_{e}+$ $n_{u}+2$. Note that the definition and the dimension of $q$ are different from those considered in Section 3.1. By following similar lines as in Section 3.1, we obtain the following hybrid model

$$
\begin{array}{cc}
\dot{q}=F(q, \xi) & q \in \mathcal{C}_{y} \cap \mathcal{C}_{u} \\
q^{+} \in G(q) & q \in \mathcal{D}_{y} \cup \mathcal{D}_{u},
\end{array}
$$

where the flow map $F(q, \xi)$ is given by

$$
F(q, \xi):=\left(f(q, \xi), g_{y}(q, \xi), g_{c}(q, \xi), 0,1,1\right)
$$

where the vector of exogenous inputs $\xi$ is defined as $\xi:=\left(w, d_{y}, \hat{d}_{u}, \dot{d}_{y}\right) \in \mathbb{R}^{n_{\xi}}$ with $\hat{d}_{u}$ denotes the value of $d_{u}$ at the transmission instant $t_{i}^{u}$. Because of the change of the definition of the samplinginduced error $e_{c}$, we obtain $f(q, \xi)=\left(f_{p}\left(x_{p}, g_{c}\left(x_{c}+\right.\right.\right.$ $\left.\left.\left.e_{c}, g_{p}\left(x_{p}, d_{y}\right)+e_{y}, \hat{d}_{u}\right), w\right), f_{c}\left(x_{c}, g_{p}\left(x_{p}, d_{y}\right)+e_{y}\right)\right)$, $g_{y}(q, \xi)=-\frac{\partial}{\partial x_{p}} g_{p}\left(x_{p}, d_{y}\right) f_{p}\left(x_{p}, g_{c}\left(x_{c}+e_{c}, g_{p}\left(x_{p}, d_{y}\right)+\right.\right.$ $\left.\left.e_{y}, \hat{d}_{u}\right), w\right)-\frac{\partial}{\partial d_{y}} g_{p}\left(x_{p}, d_{y}\right) \dot{d}_{y}$ and $g_{c}(q, \xi)=-f_{c}\left(x_{c}\right.$, $\left.g_{p}\left(x_{p}, d_{y}\right)+e_{y}\right)$. Note that the time derivative $\dot{d}_{u}$ does not appear in the dynamics $g_{c}$ of the sampling error $e_{c}$ 
contrary to Section 3. The jump map $G$ is defined as

$$
G(q):=\left\{\begin{array}{cl}
\left\{\left(x, 0, e_{c}, \hat{u}, 0, \tau_{u}\right)\right\} & q \in \mathcal{D}_{y} \backslash \mathcal{D}_{u} \\
\left\{\left(x, e_{y}, 0, u, \tau_{y}, 0\right)\right\} & q \in \mathcal{D}_{u} \backslash \mathcal{D}_{y} \\
\left\{\left(x, 0, e_{c}, \hat{u}, 0, \tau_{u}\right),\right. & \\
\left.\left(x, e_{y}, 0, u, \tau_{y}, 0\right)\right\} & q \in \mathcal{D}_{y} \cap \mathcal{D}_{u} .
\end{array}\right.
$$

The sets $\mathcal{C}_{y}, \mathcal{D}_{y}$ are defined according to the triggering condition for the plant measurements and the sets $\mathcal{C}_{u}, \mathcal{D}_{u}$ are constructed based on the triggering condition for the control input, which are given below. Solutions to system (19) flow on $\mathcal{C}_{y} \cap \mathcal{C}_{u}$, which corresponds to the region of the state space where both triggering conditions are not satisfied. When only the triggering condition involving the plant measurements or only the one involving the control input is verified, i.e. $q \in \mathcal{D}_{y} \backslash \mathcal{D}_{u}$ or $q \in \mathcal{D}_{u} \backslash \mathcal{D}_{y}$ respectively, the system experiences a jump according to (21). When both triggering conditions are satisfied at the same instant, i.e. $q \in \mathcal{D}_{y} \cap \mathcal{D}_{u}$, solutions experience two successive jumps, in view of (21). This modeling choice is justified by the fact that it ensures that the jump map $G$ is outer semicontinuous (see Definition 5.9 in [20]), as shown in [39], which is one of the hybrid basic conditions (see Assumption 6.5 in [20]). This would not be the case if we would define $G(q)$ as $\{(x, 0,0,0,0)\}$ when $q \in \mathcal{D}_{y} \cap \mathcal{D}_{u}$.

\subsection{Assumptions}

We make the following assumption on system (19). In contrast with Assumption 1, we need to introduce two functions $W_{y}$ and $W_{c}$ for the sampling-induced errors, and not a single one.

Assumption 2 There exist locally Lipschitz functions $V: \mathbb{R}^{n_{x}} \rightarrow \mathbb{R}_{\geq 0}, W_{y}: \mathbb{R}^{n_{y}} \rightarrow \mathbb{R}_{\geq 0}, W_{c}:$ $\mathbb{R}^{n_{c}} \rightarrow \mathbb{R}_{\geq 0}$ with $W_{y}, W_{c}$ positive definite, continuous functions $H_{y}, H_{c}: \mathbb{R}^{n_{x}+n_{\xi}} \rightarrow \mathbb{R}_{\geq 0}$, real numbers $L_{y_{1}}, L_{y_{2}}, L_{u_{1}}, L_{u_{2}} \geq 0, \gamma_{y}, \gamma_{c}>0, \eta \geq 0, \underline{\alpha}, \bar{\alpha} \in \mathcal{K}_{\infty}, a$ continuous function $\alpha_{s}: \mathbb{R}^{n_{x}+n_{\xi}} \rightarrow \mathbb{R}$, and continuous, functions $\delta_{y}: \mathbb{R}^{n_{y}} \rightarrow \mathbb{R}_{\geq 0}, \delta_{c}: \mathbb{R}^{n_{c}} \rightarrow \mathbb{R}_{\geq 0}$ such that

(i) for all $x \in \mathbb{R}^{n_{x}}$

$$
\underline{\alpha}(|x|) \leq V(x) \leq \bar{\alpha}(|x|)
$$

(ii) for almost all $x \in \mathbb{R}^{n_{x}}$ and all $(e, \xi) \in \mathbb{R}^{n_{e}+n_{\xi}}$

$$
\begin{aligned}
\langle\nabla V(x), f(x, e, \xi)\rangle \leq & -\alpha_{s}(x, \xi)-H_{y}^{2}(x, \xi) \\
& -H_{c}^{2}(x, \xi)-\delta_{y}(y)-\delta_{c}\left(x_{c}\right) \\
& +\gamma_{y}^{2} W_{y}^{2}\left(e_{y}\right)+\gamma_{c}^{2} W_{c}^{2}\left(e_{c}\right)
\end{aligned}
$$

(iii) for almost all $e \in \mathbb{R}^{n_{e}}$ and all $(x, \xi) \in \mathbb{R}^{n_{x}+n_{\xi}}$

$$
\begin{aligned}
\left\langle\nabla W_{y}\left(e_{y}\right), g_{y}(x, e, \xi)\right\rangle \leq & L_{y_{1}} W_{y}\left(e_{y}\right)+L_{y_{2}} W_{c}\left(e_{c}\right) \\
& +H_{y}(x, \xi) \\
\left\langle\nabla W_{c}\left(e_{c}\right), g_{c}(x, e, \xi)\right\rangle \leq & L_{u_{1}} W_{c}\left(e_{c}\right)+L_{u_{2}} W_{y}\left(e_{y}\right) \\
& +H_{c}(x, \xi) .
\end{aligned}
$$

The interpretation of item (ii) of Assumption 2 depends on the function $\alpha_{s}$ in a similar manner as we have explained after Assumption 1. We also impose item (iii) in Assumption 2 on the growth of the sampling errors $e_{y}$ and $e_{c}$. These conditions are formulated in terms of an LMI for linear systems in Section 5. We note that the dynamics of the functions $W_{y}$ and $W_{c}$ depend on both $e_{y}$ and $e_{c}$, which indicates that a mutual interaction between the growth of the sampling induced errors might exist, see Section 6 for an example.

\subsection{Event-triggering conditions}

To synthesize asynchronous event-triggering conditions, we need to carefully handle the possible mutual effect of each sampling-induced error on each other, which is reflected by (24). We propose to transmit the plant output to the controller when the condition below is verified

$$
\left(\gamma_{y}^{2}+\frac{L_{u_{2}}^{2}}{\kappa_{u}}\right) W_{y}^{2}\left(e_{y}\right) \geq \delta_{y}(y) \text { and } \tau_{y} \geq T_{y}
$$

where $\gamma_{y}, L_{u_{2}}, W_{y}, \delta_{y}$ come from Assumption $2, \kappa_{u}$ is any strictly positive constant (we explain how to tune it in the sequel), and $T_{y}>0$ is given below. We recall that $\tau_{y}$ is one of the timers introduced in Section 4.1. The stability analysis provided in the Appendix suggests to define the event-triggering part of the mechanism as in (25) and not by $\gamma_{y}^{2} W_{y}^{2}\left(e_{y}\right) \geq \delta_{y}(y)$, which is another substantial difference with the synchronous case. In view of (25), two successive transmissions of $y$ cannot occur before $T_{y}$ units of time have elapsed.

Similarly, we update the controller state $\hat{x}_{c}$ and the control input $\hat{u}$ when the following triggering condition is satisfied

$$
\left(\gamma_{c}^{2}+\frac{L_{y_{2}}^{2}}{\kappa_{y}}\right) W_{c}^{2}\left(e_{c}\right) \geq \delta_{c}\left(x_{c}\right) \text { and } \tau_{u} \geq T_{u}
$$

where $\gamma_{c}, L_{y_{2}}, W_{c}, \delta_{c}$ come from Assumption $2, \kappa_{y}$ is any strictly positive constant, and $T_{u}>0$ is given below. Note that (26) ensures that the inter-transmission times of the controller state are lower bounded by $T_{u}$. 
The constants $T_{y}$ and $T_{u}$ are selected such that $T_{y}<\mathcal{T}_{y}\left(\gamma_{y}, L_{y_{1}}, L_{u_{2}}, \kappa_{y}\right)$ and $T_{u}<\mathcal{T}_{u}\left(\gamma_{c}, L_{u_{1}}, L_{y_{2}}, \kappa_{u}\right)$, where

$$
\begin{gathered}
\mathcal{T}_{y}\left(\gamma_{y}, L_{y_{1}}, L_{u_{2}}, \kappa_{y}\right):= \begin{cases}\frac{1}{L_{y_{1}} r_{y}} \arctan \left(r_{y}\right) & \Gamma_{y}>L_{y_{1}} \\
\frac{1}{L_{y_{1}}} & \Gamma_{y}=L_{y_{1}} \\
\frac{1}{L_{y_{1}} r_{y}} \operatorname{arctanh}\left(r_{y}\right) & \Gamma_{y}<L_{y_{1}}\end{cases} \\
\mathcal{T}_{u}\left(\gamma_{c}, L_{u_{1}}, L_{y_{2}}, \kappa_{u}\right):= \begin{cases}\frac{1}{L_{u_{1}} r_{u}} \arctan \left(r_{u}\right) & \Gamma_{u}>L_{u_{1}} \\
\frac{1}{L_{u_{1}}} & \Gamma_{u}=L_{u_{1}} \\
\frac{1}{L_{u_{1}} r_{u}} \operatorname{arctanh}\left(r_{u}\right) & \Gamma_{u}<L_{u_{1}}\end{cases}
\end{gathered}
$$

with $r_{y}:=\sqrt{\left|\left(\frac{\Gamma_{y}}{L_{y_{1}}}\right)^{2}-1\right|}, r_{u}:=\sqrt{\left|\left(\frac{\Gamma_{u}}{L_{u_{1}}}\right)^{2}-1\right|}, \Gamma_{y}:=$ $\sqrt{\left(1+\kappa_{y}\right)\left(\gamma_{y}^{2}+\frac{L_{u_{2}}^{2}}{\kappa_{u}}\right)}, \Gamma_{u}:=\sqrt{\left(1+\kappa_{u}\right)\left(\gamma_{c}^{2}+\frac{L_{y_{2}}^{2}}{\kappa_{y}}\right)}$. The constants $\kappa_{y}$ and $\kappa_{u}$ offer a trade-off between $\mathcal{T}_{y}$ and $\mathcal{T}_{u}$ (we omit the arguments of $\mathcal{T}_{y}$ and $\mathcal{T}_{u}$ in the sequel for the sake of convenience). Indeed, reducing $\kappa_{y}$ generates a decrease of $\Gamma_{y}$, and therefore an increase of $\mathcal{T}_{y}$. On the other hand, this also leads to an increase of $\Gamma_{u}$ and thus a decrease of $\mathcal{T}_{u}$. The selection of the pair $\left(\kappa_{y}, \kappa_{u}\right)$ seems to be non-trivial and is out of the scope of this paper, noting that these constants also appear in the event-triggering conditions in view of (25) and (26).

The flow and the jump sets in (19) are now given by

$$
\begin{aligned}
& \mathcal{C}_{y}:=\left\{q:\left(\gamma_{y}^{2}+\frac{L_{u_{2}}^{2}}{\kappa_{u}}\right) W_{y}^{2}\left(e_{y}\right) \leq \delta_{y}(y) \text { or } \tau_{y} \in\left[0, T_{y}\right]\right\} \\
& \mathcal{C}_{u}:=\left\{q:\left(\gamma_{c}^{2}+\frac{L_{y_{2}}^{2}}{\kappa_{y}}\right) W_{c}^{2}\left(e_{c}\right) \leq \delta_{c}\left(x_{c}\right) \text { or } \tau_{u} \in\left[0, T_{u}\right]\right\} \\
& \mathcal{D}_{y}:=\left\{q:\left(\gamma_{y}^{2}+\frac{L_{u_{2}}^{2}}{\kappa_{u}}\right) W_{y}^{2}\left(e_{y}\right) \geq \delta_{y}(y) \text { and } \tau_{y} \geq T_{y}\right\} \\
& \mathcal{D}_{u}:=\left\{q:\left(\gamma_{c}^{2}+\frac{L_{y_{2}}^{2}}{\kappa_{y}}\right) W_{c}^{2}\left(e_{c}\right) \geq \delta_{c}\left(x_{c}\right) \text { and } \tau_{u} \geq T_{u}\right\} .
\end{aligned}
$$

We note that both $\hat{y}$ and $\hat{x}_{c}$ may be updated at the same time instant, which prevents the existence of a dwelltime for the overall system (19), like in other works on asynchronous event-triggered control, see [18], [42].

\section{$4.4 \quad \mathcal{L}_{p}$ Stability}

Due to space limitations, we only focus in this section on $\mathcal{L}_{p}$ stability; similar results can be derived to ensure an ISS property like in Section 3.4. The following theorem ensures an $\mathcal{L}_{p}$ stability property for the closed-loop system (19) under the event-triggering mechanism (28). The arguments of $\mathcal{T}_{y}, \mathcal{T}_{u}$ are omitted in the sequel for convenience.

Theorem 3 Consider system (19) with the flow and the jump sets in (28) and the output $z=h(x, \xi)$, where the constants $T_{y}, T_{u}$ are such that $T_{y} \in\left(0, \mathcal{T}_{y}\right)$ and $T_{u} \in$ $\left(0, \mathcal{T}_{u}\right)$. Suppose that Assumption 2 holds with $\alpha_{s}(x, \xi)=$ $|z|^{p}-\eta^{p}|\xi|^{p}$, where $p \in \mathbb{Z}_{>0}$ and $\eta \geq 0$. Then, system (19) is $\mathcal{L}_{p}$ stable from $\xi$ to $z$ with an $\overline{\mathcal{L}}_{p}$ gain less than or equal to $\eta$.

\subsection{Time-triggered control}

The transmission laws developed in Section 4.3 can be applied to the case where transmissions are timetriggered. For instance, asynchronous time-triggered implementations directly follows from (28) by taking

$$
\begin{array}{ll}
\mathcal{C}_{y}=\left\{q: \tau_{y} \in\left[0, T_{y}\right]\right\}, & \mathcal{D}_{y}=\left\{q: \tau_{y} \in\left[\epsilon_{y}, T_{y}\right]\right\} \\
\mathcal{C}_{u}=\left\{q: \tau_{u} \in\left[0, T_{u}\right]\right\}, & \mathcal{D}_{u}=\left\{q: \tau_{u} \in\left[\epsilon_{u}, T_{u}\right]\right\}
\end{array}
$$

where $\epsilon_{y} \in\left(0, T_{y}\right], \epsilon_{u} \in\left(0, T_{u}\right]$ are introduced to prevent Zeno behaviour, and $T_{y}, T_{u}$ are strictly smaller than $\mathcal{T}_{y}$, $\mathcal{T}_{u}$ defined in (27). When $\epsilon_{y}=T_{y}$ and $\epsilon_{u}=T_{u}$, the sets in (28) lead to periodic and asynchronous transmissions of the output measurement and of the control input, respectively. Then the conclusion of Theorem 3 holds, by following similar lines as in the proof of Theorem 3, when Assumption 2 is verified. Note that in the case of time-triggered control, the functions $\delta_{y}, \delta_{c}$ in $(23)$ are not needed and thus Assumption 2 can be relaxed.

Remark 2 The time-triggered implementation of the case where the output measurement and the control input are transmitted synchronously can be realized in a similar way by modifying the flow and the jump sets in (15) to be $\mathcal{C}=\{q: \tau \in[0, T]\}$ and $\mathcal{D}=\{q: \tau \in[\epsilon, T]\}$, where $\epsilon \in(0, T]$ and $T$ is upper bounded by $\mathcal{T}$ defined in (14).

\section{Case study: linear time-invariant systems}

We apply the results to LTI systems. Due to space constraints, we focus on $\mathcal{L}_{2}$ stabilization under asynchronous transmissions. The results for the cases of synchronous implementation and ISS stabilization similarly follows. Consider the LTI plant model

$$
\dot{x}_{p}=A_{p} x_{p}+B_{p} u+E_{p} w, \quad y=C_{p} x_{p}+d_{y},
$$

where $x_{p} \in \mathbb{R}^{n_{p}}, u \in \mathbb{R}^{n_{u}}, w \in \mathbb{R}^{n_{w}}, y \in \mathbb{R}^{n_{y}}, d_{y} \in$ $\mathbb{R}^{n_{y}}$ and $A_{p}, B_{p}, E_{p}, C_{p}$ are matrices of appropriate dimensions, with $\left(A_{p}, B_{p}\right)$ stabilizable and $\left(A_{p}, C_{p}\right)$ detectable; . The plant is stabilized by the following feedback law in the absence of exogenous inputs

$$
\dot{x}_{c}=A_{c} x_{c}+B_{c} y \quad u=C_{c} x_{c}+D_{c} y+d_{u},
$$

where $x_{c} \in \mathbb{R}^{n_{c}}, d_{u} \in \mathbb{R}^{n_{u}}$, and $A_{c}, B_{c}, C_{c}, D_{c}$ are matrices of appropriate dimensions. We then take into account the sampling as in Section 3.1. We obtain the hy- 


$$
\begin{aligned}
& \left(\begin{array}{cccc}
\Sigma & \star & \star & \star \\
\mathcal{B}_{1}^{T} \boldsymbol{P} & -\boldsymbol{\mu}_{y} \mathbb{I}_{n_{y}} & \star & \star \\
\mathcal{M}_{1}^{T} \boldsymbol{P} & 0 & -\boldsymbol{\mu}_{u} \mathbb{I}_{n_{c}} & \star \\
\mathcal{E}_{1}^{T} \boldsymbol{P}+\boldsymbol{\varepsilon}_{y} D_{y}^{T} C_{y}+D_{z}^{T} C_{z}+\boldsymbol{\lambda}_{y}^{2} \mathcal{E}_{2}^{T} \mathcal{A}_{2}+\boldsymbol{\lambda}_{u}^{2} \mathcal{E}_{3}^{T} \mathcal{A}_{3} & 0 & 0 & D_{z}^{T} D_{z}+\boldsymbol{\lambda}_{y}^{2} \mathcal{E}_{2}^{T} \mathcal{E}_{2}+\boldsymbol{\lambda}_{u}^{2} \mathcal{E}_{3}^{T} \mathcal{E}_{3}+\boldsymbol{\varepsilon}_{y} D_{y}^{T} D_{y}-\boldsymbol{\vartheta} \mathbb{I}_{n_{\xi}}
\end{array}\right)<0 \\
& \Sigma:=\mathcal{A}_{1}^{T} \boldsymbol{P}+\boldsymbol{P} \mathcal{A}_{1}+C_{z}^{T} C_{z}+\lambda_{y}^{2} \mathcal{A}_{2}^{T} \mathcal{A}_{2}+\lambda_{u}^{2} \mathcal{A}_{3}^{T} \mathcal{A}_{3}+\varepsilon_{y} C_{y}^{T} C_{y}+\varepsilon_{c} C_{u}^{T} C_{u} \\
& C_{y}:=\left(C_{p}, 0\right), \quad C_{u}:=\left(0, C_{c}\right), \quad D_{y}:=(0,1,0,0) \text {. }
\end{aligned}
$$

brid model below (recall that $\left.q=\left(x, e_{y}, e_{c}, \hat{u}, \tau_{y}, \tau_{u}\right)\right)$

$$
\dot{q}=\left(\begin{array}{c}
\mathcal{A}_{1} x+\mathcal{B}_{1} e_{y}+\mathcal{M}_{1} e_{c}+\mathcal{E}_{1} \xi \\
\mathcal{A}_{2} x+\mathcal{B}_{2} e_{y}+\mathcal{M}_{2} e_{c}+\mathcal{E}_{2} \xi \\
\mathcal{A}_{3} x+\mathcal{B}_{3} e_{y}+\mathcal{E}_{3} \xi \\
0 \\
1 \\
1
\end{array}\right) q \in \mathcal{C}_{y} \cap \mathcal{C}_{u}
$$

with $G(q)$ defined in (21), $\mathcal{A}_{1}:=\left[\begin{array}{cc}A_{p}+B_{p} D_{c} C_{p} & B_{p} C_{c} \\ B_{c} C_{p} & A_{c}\end{array}\right]$, $\mathcal{B}_{1}:=\left[\begin{array}{c}B_{p} D_{c} \\ B_{c}\end{array}\right], \quad \mathcal{M}_{1}:=\left[\begin{array}{c}B_{p} C_{c} \\ 0\end{array}\right], \quad \mathcal{E}_{1}:=\left[\begin{array}{cccc}E_{p} & B_{p} D_{c} & B_{p} & 0 \\ 0 & B_{c} & 0 & 0\end{array}\right]$, $\mathcal{A}_{2}:=-C_{p}\left[\begin{array}{ll}A_{p}+B_{p} D_{c} C_{p} & B_{p} C_{c}\end{array}\right], \quad \mathcal{B}_{2}:=-C_{p} B_{p} D_{c}$, $\mathcal{M}_{2}:=-C_{p} B_{p} C_{c}, \quad \mathcal{E}_{2}:=\left[\begin{array}{lll}-C_{p} E_{p} & -C_{p} B_{p} D_{c}-C_{p} B_{p}-1\end{array}\right]$,

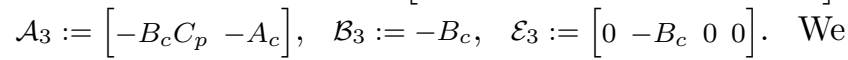
consider the following controlled output

$$
z=C_{z} x+D_{z} \xi
$$

where $C_{z}, D_{z}$ are matrices of appropriate dimensions.

The proposition below states that the satisfaction of a linear matrix inequality ensures that Assumption 2 holds, which in turn implies that the conclusions of Theorem 3 hold. We use boldface symbols in the LMI to emphasize the decision variables.

Proposition 1 Consider system (32). Suppose that there exist $\varepsilon_{y}, \varepsilon_{c}, \mu_{y}, \mu_{u}, \lambda_{y}, \lambda_{u}, \vartheta>0$ and a positive definite symmetric real matrix $P$ such that (34) holds. Take $V(x)=x^{T} P x, W_{y}\left(e_{y}\right)=\lambda_{y}\left|e_{y}\right|$ and $W_{c}\left(e_{c}\right)=\lambda_{u}\left|e_{c}\right|$, for all $x \in \mathbb{R}^{n_{x}},\left(e_{y}, e_{c}\right) \in \mathbb{R}^{n_{e}}$. Then Assumption 2 holds with $\underline{\alpha}(s)=\lambda_{\min }(P) s^{2}, \bar{\alpha}(s)=\lambda_{\max }(P) s^{2}$, $\alpha_{s}(x, \xi)=|z|^{2}-\eta^{2}|\xi|^{2}$ with $\eta=\sqrt{\vartheta}$ for $s \geq 0, H_{y}(x, \xi)=$ $\lambda_{y}\left|\mathcal{A}_{2} x+\mathcal{E}_{2} \xi\right|, H_{c}(x, \xi)=\lambda_{u}\left|\mathcal{A}_{3} x+\mathcal{E}_{3} \xi\right|, \delta_{y}(y)=\varepsilon_{y}|y|^{2}$, $\delta_{c}\left(x_{c}\right)=\varepsilon_{c}\left|x_{c}\right|^{2}, L_{y_{1}}=\left|\mathcal{B}_{2}\right|, L_{y_{2}}=\frac{\lambda_{y}}{\lambda_{u}}\left|\mathcal{M}_{2}\right|, L_{u_{1}}=0$, $L_{u_{2}}=\frac{\lambda_{u}}{\lambda_{y}}\left|\mathcal{B}_{3}\right|, \gamma_{y}=\frac{\sqrt{\mu_{y}}}{\lambda_{y}}, \gamma_{c}=\frac{\sqrt{\mu_{u}}}{\lambda_{u}}$.
Proposition 1 provides a systematic way to verify the required conditions in Theorem 3. Note that, since the lefthand side of (34) is a symmetric real matrix, a necessary condition to guarantee the feasibility of LMI (34) is that $D_{z}^{T} D_{z}+\boldsymbol{\lambda}_{y}^{2} \mathcal{E}_{2}^{T} \mathcal{E}_{2}+\boldsymbol{\lambda}_{u}^{2} \mathcal{E}_{3}^{T} \mathcal{E}_{3}+\varepsilon_{y} D_{y}^{T} D_{y}-\boldsymbol{\vartheta} \mathbb{I}_{n_{\xi}} \leq 0$. This condition can be always verified by selecting $\lambda_{y}, \lambda_{u}, \varepsilon_{y}$ sufficiently small and by taking $\vartheta$ sufficiently large. However, when $\lambda_{y}, \lambda_{u}$ are small, $\gamma_{y}=\frac{\sqrt{\mu_{y}}}{\lambda_{y}}$ and $\gamma_{c}=\frac{\sqrt{\mu_{u}}}{\lambda_{u}}$ become large, which leads to a smaller upper bound on the MATI, see (14). Moreover, when $\varepsilon_{y}$ is small and $\gamma_{y}$ increases, the event-triggering rule $\left(\gamma_{y}^{2}+\frac{L_{u_{2}}^{2}}{\kappa_{u}}\right) W_{y}^{2}\left(e_{y}\right) \leq$ $\varepsilon_{y}|y|^{2}$ may be quickly violated, which would lead to smaller inter-transmission times for the plant output. Hence, $\lambda_{y}, \lambda_{u}, \varepsilon_{y}$ can be used as tuning parameters to make a tradeoff between the estimated $\mathcal{L}_{2}$ gain $\eta$ on one side and the MATI and the amount of transmissions generated by the event-triggering mechanism on the other side.

Remark 3 In the absence of sampling, condition (34) reduces to the existence of a real matrix $P=P^{T}>0$ and a scalar $\vartheta^{*}>0$ such that

$$
\left(\begin{array}{cc}
\mathcal{A}_{1}^{T} \boldsymbol{P}+\boldsymbol{P} \mathcal{A}_{1}+C_{z}^{T} C_{z} & \star \\
\mathcal{E}_{1}^{T} \boldsymbol{P}+D_{z}^{T} C_{z} & D_{z}^{T} D_{z}-\boldsymbol{\vartheta}^{*} \mathbb{I}_{n_{\xi}}
\end{array}\right)<0,
$$

where $\eta^{*}=\sqrt{\vartheta^{*}}$ is an estimate of the $\mathcal{L}_{2}$ gain in the absence of a network. Note that LMI (35) is equivalent to the standard $\mathcal{L}_{2}$ gain $/ \mathcal{H}_{\infty}$ LMI for LTI systems, see Corollary 2.19 and Proposition 2.24 in [40], which is always feasible when system (30) is stabilizable and detectable as assumed here. The feasibility of LMI (35) implies that, by applying the Schur complement, LMI (34) is feasible for $\lambda_{y}, \lambda_{u}, \varepsilon_{y}, \varepsilon_{c}$ sufficiently small and for $\mu_{y}, \mu_{u}$ sufficiently large. 


$$
\begin{aligned}
& \mathcal{A}_{1}:=\left[\begin{array}{cc}
A & B K \\
M C & A-M C+B K
\end{array}\right], \quad \mathcal{B}_{1}:=\left[\begin{array}{c}
0 \\
M
\end{array}\right], \mathcal{M}_{1}:=\left[\begin{array}{l}
B K \\
B K
\end{array}\right], \mathcal{E}_{1}:=\left[\begin{array}{cc}
E & B \\
0 & B
\end{array}\right], \psi\left(y, e_{y}\right):=\left[\begin{array}{c}
\phi(y+e)-\phi(y) \\
0
\end{array}\right] \\
& \mathcal{A}_{2}:=[-M C-(A-M C+B K)], \mathcal{B}_{2}:=-M, \quad \mathcal{M}_{2}:=-B K, \quad \mathcal{E}_{2}:=\left[\begin{array}{ll}
0 & -B
\end{array}\right]
\end{aligned}
$$

\section{Illustrative example}

\subsection{Model of a single-link robot arm}

Consider the dynamics of a single-link robot arm

$$
\begin{aligned}
& \dot{x}_{p 1}=x_{p 2}, \quad \dot{x}_{p 2}=-\sin \left(x_{p 1}\right)+u+w, \\
& y \quad=x_{p 1},
\end{aligned}
$$

where $x_{p 1}$ denotes the angle, $x_{p 2}$ the rotational velocity, $u$ the input torque and $w$ is the external disturbance. We ignore measurement noises to simplify the presentation only. The system can be written as

$$
\dot{x}_{p}=A x_{p}+B u-\phi(y)+E w \quad y=C x_{p},
$$

where $x_{p}:=\left(x_{p 1}, x_{p 2}\right), A=\left[\begin{array}{ll}0 & 1 \\ 0 & 0\end{array}\right], B=\left[\begin{array}{l}0 \\ 1\end{array}\right], C=$ $\left[\begin{array}{l}1 \\ 0\end{array}\right]^{T}, \phi(y)=\left[\begin{array}{c}0 \\ \sin (y)\end{array}\right], E=\left[\begin{array}{l}0 \\ 1\end{array}\right]$. In order to stabilize system (37), we first construct a state feedback controller of the form $u=K x_{p}+B^{T} \phi(y)+d_{u}$, where $d_{u} \in \mathbb{R}$ is the noise on the control input. We design the gain $K$ such that the eigenvalues of $A+B K$ are -1 and -2 (which is possible since the pair $(A, B)$ is controllable). Hence, the gain $K$ is selected to be $K=\left[\begin{array}{ll}-2 & -3\end{array}\right]$. Next, since only the measurement of $y$ is available, we construct a state-observer of the following form

$$
\begin{aligned}
\dot{x}_{c} & =A x_{c}+B u-\phi(y)+M\left(y-C x_{c}\right) \\
& =(A-M C) x_{c}+B u-\phi(y)+M y,
\end{aligned}
$$

where $x_{c} \in \mathbb{R}^{2}$ is the estimated state and $M$ is the observer gain matrix. We design the gain matrix $M$ such that the eigenvalues of $A-M C$ are -5 and -6 (which is possible since the pair $(A, C)$ is observable), which leads to $M=\left[\begin{array}{ll}11 & 30\end{array}\right]^{T}$. We now take into account the effect of the network and we study the two implementation scenarios. We focus on the $\mathcal{L}_{2}$ stability. We consider the measured output $y$ as the controlled output, i.e. $z=y=x_{p 1}$.

\subsection{Verification of Assumption 2}

We consider here the scenario where the output measurement and the controller state are asynchronously sampled by using two different triggering conditions. Hence, as explained in Section 4, we define the network-induced error as $e=\left(e_{y}, e_{c}\right)$ with $e_{y}=\hat{y}-y, e_{c}=\hat{x}_{c}-x_{c}$, and the vector of exogenous inputs $\xi$ is defined as $\xi=\left(w, d_{u}\right)$. Then, we obtain

$$
\begin{aligned}
& \dot{x}=\mathcal{A}_{1} x+\mathcal{B}_{1} e_{y}+\mathcal{M}_{1} e_{c}+\mathcal{E}_{1} \xi+\psi\left(y, e_{y}\right)=f(q, \xi) \\
& \dot{e}_{y}=-x_{p 2} \quad=g_{y}(q, \xi) \\
& \dot{e}_{c}=\mathcal{A}_{2} x+\mathcal{B}_{2} e_{y}+\mathcal{M}_{2} e_{c}+\mathcal{E}_{2} \xi \quad=g_{c}(q, \xi)
\end{aligned}
$$

with the system matrices and $\psi$ as defined in (41).

Now we verify that Assumption 2 holds to apply the result of Theorem 4.4. Let $W_{y}\left(e_{y}\right):=\lambda_{y}\left|e_{y}\right|$ and $W_{c}\left(e_{c}\right):=\lambda_{u}\left|e_{c}\right|$ for all $e \in \mathbb{R}^{n_{e}}$ and $\lambda_{y}, \lambda_{u}>0$. Consequently, for almost all $e \in \mathbb{R}^{n_{e}}$ and all $x \in \mathbb{R}^{n_{x}}$, $\left\langle\nabla W_{y}\left(e_{y}\right), g_{y}(q, \xi)\right\rangle \leq \lambda_{y}\left|x_{p 2}\right|$ and $\left\langle\nabla W_{c}\left(e_{c}\right), g_{c}(q, \xi)\right\rangle \leq$ $\lambda_{u}\left|\mathcal{A}_{2} x+\mathcal{E}_{2} \xi\right|+\lambda_{u}\left|\overline{\mathcal{B}}_{2}\right|\left|e_{y}\right|+\lambda_{u}\left|\mathcal{M}_{2}\right|\left|e_{c}\right|$. Hence, condition (24) holds with $H_{y}(x, \xi)=\lambda_{y}\left|x_{p 2}\right|, L_{y 1}=L_{y 2}=0$, $H_{c}(x, \xi)=\lambda_{u}\left|\mathcal{A}_{2} x+\mathcal{E}_{2} \xi\right|, L_{u_{1}}=\left|\mathcal{M}_{2}\right|$ and $L_{u_{2}}=$ $\frac{\lambda_{u}}{\lambda_{y}}\left|\mathcal{B}_{2}\right|$. Let $V(x)=x^{T} P x$, where $P$ is a real positive definite symmetric matrix such that $\mathcal{A}_{1}^{T} P+P \mathcal{A}_{1}=-Q$ and $Q$ is a real positive definite and symmetric matrix. For all $(x, e) \in \mathbb{R}^{n_{x}+n_{e}}$,

$$
\begin{aligned}
\langle\nabla V(x), f(x, e, \xi)\rangle \leq & -\lambda_{\min }(Q)|x|^{2}+2\left|P \mathcal{B}_{1}\|x\| e_{y}\right| \\
& +2\left|P \mathcal{M}_{1}\right|\left|x\left\|e_{c}|+2| P \mathcal{E}_{1}|| x\right\| \xi\right| \\
& +2\left|P\|x\| \psi\left(y, e_{y}\right)\right|
\end{aligned}
$$

We have that $\left|\psi\left(y, e_{y}\right)\right|=\left|\phi\left(y+e_{y}\right)-\phi(y)\right|=\mid \sin (y+$ $\left.e_{y}\right)-\sin (y)|\leq| e_{y} \mid$. By using the fact that $2\left(\left|P \mathcal{B}_{1}\right|+\right.$ $|P|)|x|\left|e_{y}\right| \leq \nu_{1}|x|^{2}+\frac{\left(\left|P \mathcal{B}_{1}\right|+|P|\right)^{2}}{\nu_{1}}\left|e_{y}\right|^{2}, 2\left|P \mathcal{M}_{1}\right||x|\left|e_{c}\right| \leq$ $\nu_{2}|x|^{2}+\frac{\left|P \mathcal{M}_{1}\right|^{2}}{\nu_{2}}\left|e_{c}\right|^{2}, 2\left|P \mathcal{E}_{1}\right||x||\xi| \leq \nu_{3}|x|^{2}+\frac{\left|P \mathcal{E}_{1}\right|^{2}}{\nu_{3}}|\xi|^{2}$, $|z| \leq|x|,\left|x_{c}\right| \leq|x|$, and by adding and subtracting the terms $|x|^{2}+\varepsilon_{c}|x|^{2}$, it holds that, for $\varepsilon_{y}, \varepsilon_{c} \geq 0$

$$
\begin{aligned}
\langle\nabla V(x), f(q, \xi)\rangle \leq & -\bar{\alpha}|x|^{2}-|z|^{2}-\varepsilon_{y}|y|^{2}-\varepsilon_{c}\left|x_{c}\right|^{2} \\
& +\frac{\left(\left|P \mathcal{B}_{1}\right|+|P|\right)^{2}}{\nu_{1}}\left|e_{y}\right|^{2}+\frac{\left|P \mathcal{M}_{1}\right|^{2}}{\nu_{2}}\left|e_{c}\right|^{2} \\
& +\frac{\left|P \mathcal{E}_{1}\right|^{2}}{\nu_{3}}|\xi|^{2}
\end{aligned}
$$

where $\bar{\alpha}:=\lambda_{\min }(Q)-\nu_{1}-\nu_{2}-\nu_{3}-\varepsilon_{y}-\varepsilon_{c}-1$. By adding and subtracting $H_{y}^{2}(x, \xi)=\lambda_{y}^{2}\left|x_{p 2}\right|^{2}$ and $H_{c}^{2}(x, \xi)=$ 
$\lambda_{u}^{2}\left|\mathcal{A}_{2} x+\mathcal{E}_{2} \xi\right|^{2} \leq 2 \lambda_{u}^{2}\left|\mathcal{A}_{2}\right|^{2}|x|^{2}+2 \lambda_{u}^{2}\left|\mathcal{E}_{2}\right|^{2}|\xi|^{2}$, we obtain

$$
\begin{aligned}
\langle\nabla V(x), f(q, \xi)\rangle \leq & -\alpha|x|^{2}-|z|^{2}-H_{y}^{2}(x, \xi)-H_{c}^{2}(x, \xi) \\
& -\varepsilon_{y}|y|^{2}-\varepsilon_{c}\left|x_{c}\right|^{2}+\gamma_{y}^{2} W_{y}^{2}\left(e_{y}\right) \\
& +\gamma_{c}^{2} W_{c}^{2}\left(e_{c}\right)+\eta^{2}|\xi|^{2}
\end{aligned}
$$

where $\alpha:=\lambda_{\min }(Q)-\nu_{1}-\nu_{2}-\nu_{3}-\varepsilon_{y}-\varepsilon_{c}-1-$ $2 \lambda_{y}^{2}-2 \lambda_{u}^{2}\left|\mathcal{A}_{2}\right|^{2}, \gamma_{y}=\sqrt{\frac{\left(\left|P \mathcal{B}_{1}\right|+|P|\right)^{2}}{\nu_{1} \lambda_{y}^{2}}}, \gamma_{c}=\sqrt{\frac{\left|P \mathcal{M}_{1}\right|^{2}}{\nu_{2} \lambda_{u}^{2}}}$ and $\eta=\sqrt{\left(\frac{\left|P \mathcal{E}_{1}\right|^{2}}{\nu_{3}}+2 \lambda_{u}^{2}\left|\mathcal{E}_{2}\right|^{2}\right)}$. Thus, by taking $Q$ such that $\lambda_{\min }(Q)>\nu_{1}+\nu_{2}+\nu_{3}+\varepsilon_{y}+\varepsilon_{c}+1+2 \lambda_{y}^{2}+2 \lambda_{u}^{2}\left|\mathcal{A}_{2}\right|^{2}$, which ensures that $\alpha>0$, condition (23) is verified with $\delta_{y}(y)=\varepsilon_{y}|y|^{2}, \delta_{c}\left(x_{c}\right)=\varepsilon_{c}\left|x_{c}\right|^{2}$ and an $\mathcal{L}_{2}$ gain less than or equal to $\eta$.

To set the design parameters $\kappa_{y}, \kappa_{u}, \nu_{1}, \nu_{2}, \nu_{3}, \varepsilon_{y}, \varepsilon_{c}, \lambda_{y}$, and $\lambda_{u}$, we use the MATLAB fmincon optimizer to minimize $\gamma_{y}+\gamma_{c}+\eta$ such that these parameters are strictly positive. We obtain $\kappa_{y}=\kappa_{u}=0.01, \nu_{1}=\nu_{2}=\nu_{3}=$ $1, \varepsilon_{y}=\varepsilon_{c}=1, \lambda_{y}=1$, and $\lambda_{u}=0.01$, which yield $L_{u_{1}}=0.0361, L_{u_{2}}=0.3195, \gamma_{y}=278.2691, \gamma_{c}=4305.8$ and $\mathcal{L}_{2}$ gain $\eta=19.3746$. By substituting in (27), we obtain $\mathcal{T}_{y}=5.62 \times 10^{-3}$ and $\mathcal{T}_{u}=3.63 \times 10^{-4}$.

\subsection{Simulation results}

We take $T_{y}=5.6 \times 10^{-3}$ and $T_{u}=3.6 \times 10^{-4}$ and we consider the random disturbance $w$ satisfies $|w(t)| \leq 0.1$ and the noise on the control input $d_{u}(t)=0.1 \sin (50 t)$ for any $t \geq 0$. We have run simulations for 100 randomly distributed initial conditions such that $|x(0,0)| \leq 100$, $e_{y}(0,0)=0, e_{c}(0,0)=(0,0)$, and $\left(\tau_{y}(0,0), \tau_{u}(0,0)\right)=$ $(0,0)$ for $10 \mathrm{~s}$. The obtained minimum and the average inter-transmission times, respectively denoted by $\tau_{\min }$ and $\tau_{\text {avg }}$, are give in Table 1.

\begin{tabular}{c|c|c|c} 
& $\begin{array}{c}\text { Guaranteed } \\
\text { dwell-time }\end{array}$ & $\tau_{\text {min }}$ & $\tau_{\text {avg }}$ \\
\hline plant output $y$ & $5.6 \times 10^{-3}$ & $5.6 \times 10^{-3}$ & $8.4 \times 10^{-3}$ \\
\hline control input $u$ & $3.6 \times 10^{-4}$ & $3.6 \times 10^{-4}$ & $67 \times 10^{-4}$
\end{tabular}

Table 1

Minimum and average inter-transmission times for the output measurement and the control input.

The generated inter-transmission times with the initial condition of the state $x(0,0)=(10,-10,-10,10)$ are shown in Figure 4. We note that the enforced constant times $T_{y}, T_{u}$ (represented by the constant lines) act as lower bounds on the inter-transmission times which justify the proposed triggering mechanism. The state trajectories of the plant and of the controller are shown in Figure 3 where we notice that the states asymptotically converge to a neighborhood of the origin as expected. We have compared the results with periodic time-triggered controllers, that is with $(29)$ and $T_{y}=5.6 \times 10^{-3}$ and $T_{u}=3.6 \times 10^{-4}$. We have noticed that the state trajectories are almost identical to those in Figure 3 and cannot be distinguished. However, as shown in Table 1, event-triggered controllers leads to reducing the average amount of transmissions by $33 \%$ for the output measurement and by $95 \%$ for the control input, which shows the advantage of the proposed event-triggering mechanism. Finally, the tradeoff curves between the $\mathcal{L}_{2}$ gain $\eta$ and $T_{y}$ and $T_{u}$ are presented in Figures 5,6 , respectively. We observe that smaller values of $\eta$ lead to smaller lower bound $T_{y}, T_{u}$ on the inter-transmission times and vice versa.

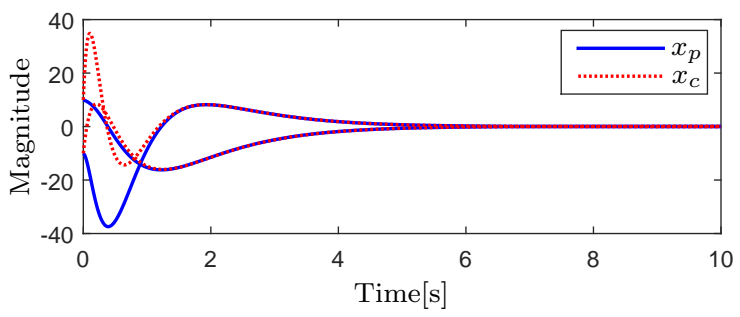

Fig. 3. State trajectories of the plant and the controller with event-triggering mechanism (28).
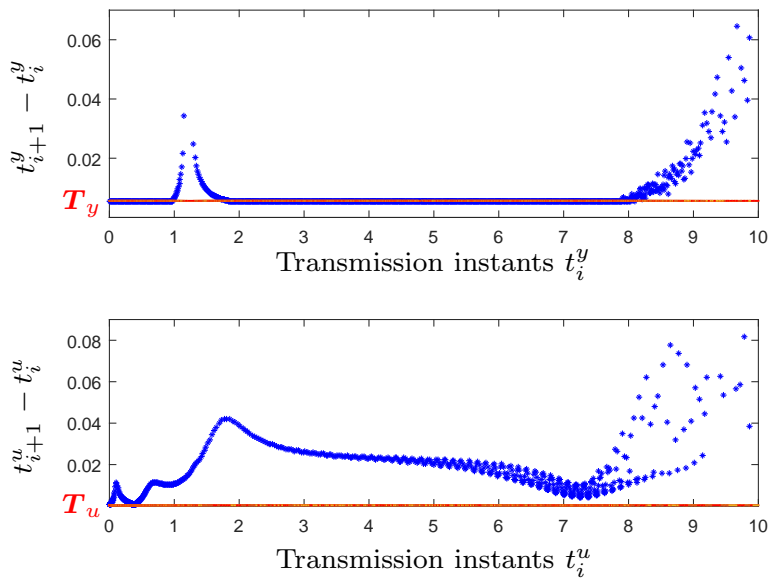

Fig. 4. Inter-transmission times for the plant output (upper plot) and for the controller state (lower plot).

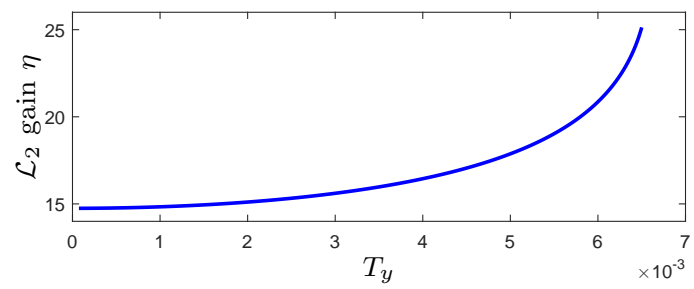

Fig. 5. Tradeoff curve between the $\mathcal{L}_{2}$ gain $\eta$ and $T_{y}$. 


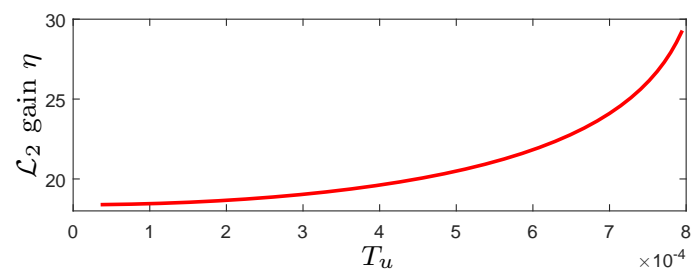

Fig. 6. Tradeoff curve between the $\mathcal{L}_{2}$ gain $\eta$ and $T_{u}$.

\section{Conclusion}

We have investigated robust stabilization of nonlinear systems using output feedback event-triggered controllers with both synchronous and asynchronous communications. The proposed techniques ensure ISS and $\mathcal{L}_{p}$ stability properties for the closed-loop system and we enforce uniform strictly positive lower bounds on the inter-transmissions times. The approach is applied to LTI systems as a particular case and has been illustrated on a physical nonlinear example.

The results presented in this paper have many natural extensions. For instance, the flexibility of the proposed mechanism can be enhanced by jointly designing the feedback law and the event-triggering condition in the case of linear systems like in [3], as opposed to the emulation approach. On the other hand, we believe that the proposed techniques can be adapted for the distributed event-triggered control of nonlinear systems. In this context, Zeno phenomenon is also a major issue and we expect that triggering laws similar to the ones presented in this paper could be synthesized to handle this issue.

\section{Appendix}

We first present some definitions and results that we use in the proofs of our main results. Since we consider locally Lipschitz Lyapunov functions (that are not necessarily differentiable everywhere), we use the generalized directional derivative of Clarke which is defined as follows. For a locally Lipschitz function $U: \mathbb{R}^{n} \rightarrow \mathbb{R}$ and

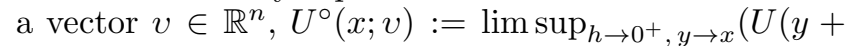
$h v)-U(y)) / h$. For a continuously differentiable function $U, U^{\circ}(x ; v)$ reduces to the standard directional derivative. We will define Lyapunov functions as the maximum of two locally Lipschitz functions and we will invoke the following result, see Lemma II.1 in [29].

Lemma 1 Consider two functions $u_{1}: \mathbb{R}^{n} \rightarrow \mathbb{R}$ and $u_{2}: \mathbb{R}^{n} \rightarrow \mathbb{R}$ that have well-defined Clarke derivatives for all $x \in \mathbb{R}^{n}$ and $v \in \mathbb{R}^{n}$. Introduce three sets $A:=$ $\left\{x: u_{1}(x)>u_{2}(x)\right\}, B:=\left\{x: u_{1}(x)<u_{2}(x)\right\}, \Gamma:=$ $\left\{x: u_{1}(x)=u_{2}(x)\right\}$. Then, for any $v \in \mathbb{R}^{n}$, the function $U(x):=\max \left\{u_{1}(x), u_{2}(x)\right\}$ satisfies $U^{\circ}(x ; v)=u_{1}^{\circ}(x ; v)$ for all $x \in A, U^{\circ}(x ; v)=u_{2}^{\circ}(x ; v)$ for all $x \in B$ and $U^{\circ}(x ; v) \leq \max \left\{u_{1}^{\circ}(x ; v), u_{2}^{\circ}(x ; v)\right\}$ for all $x \in \Gamma$.
Proof of Theorem 1. We first introduce $\zeta: \mathbb{R}_{\geq 0} \rightarrow \mathbb{R}$ the solution to the following differential equation

$$
\dot{\zeta}=-2 L \zeta-(1+\kappa) \gamma\left(\zeta^{2}+1\right) \quad \zeta(0)=\theta^{-1},
$$

where $\theta \in(0,1)$ and $\kappa>0$. We denote $\tilde{\mathcal{T}}(\theta, \kappa, \gamma, L)$ the time it takes for $\zeta$ to decrease from $\theta^{-1}$ to $\theta$. This time $\tilde{\mathcal{T}}(\theta, \kappa, \gamma, L)$ is a continuous function of $\theta, \kappa$ which is decreasing in $\theta, \kappa$. On the other hand, we note that $\tilde{\mathcal{T}}(\theta, \gamma, \kappa, L) \rightarrow \mathcal{T}(\kappa, \gamma, L)$ (where $\mathcal{T}(\kappa, \gamma, L)$ is defined in (14)) as $\theta$ tends to 0 , see Lemma 1 in [16]. As a consequence, since $T<\mathcal{T}(\kappa, \gamma, L)$, there exists $\theta$ such that $T<\widetilde{\mathcal{T}}(\theta, \kappa, \gamma, L)$. We fix the value of $\theta$.

We define for all $q \in \mathcal{C} \cup \mathcal{D}, R(q):=V(x)+$ $\max \left\{0, \gamma \zeta(\tau) W^{2}(e)\right\}$.

Let $q \in \mathcal{D}$ and $G(q):=(x, 0,0)$. We obtain, in view of (8) and the fact that $W$ is positive definite,

$$
\begin{aligned}
R(G(q)) & =V(x)+\max \left\{0, \gamma \zeta(0) W^{2}(0)\right\} \\
& =V(x) \leq R(q) .
\end{aligned}
$$

Let $q \in \mathcal{C}$ and $\xi \in \mathbb{R}^{n_{\xi}}$ and suppose that $\zeta(\tau)<0$. As a consequence, $R(q)=V(x)$ and it holds that $\tau>T$. Indeed, $\zeta(\tau)$ is strictly decreasing in $\tau$, in view of (45), and $\zeta(T)>\zeta(\tilde{\mathcal{T}})=\theta>0$ as $T<\tilde{\mathcal{T}}$. Then $\zeta(\tau)<0$ implies that $\tau>T$. Hence, $\gamma^{2} W^{2}(e) \leq \delta(y)$ in view of (15) since $q \in \mathcal{C}$. Consequently, in view of page 100 in [43], Lemma 1, Assumption 1 and the definition of $R, R^{\circ}(q ; F(q, \xi)) \leq-\alpha(|x|)+\varrho(|\xi|)$, where $F(q, \xi):=$ $(f(x, e, \xi), g(x, e, \xi), 1)$. Hence, in view of (10) and since $\alpha \in \mathcal{K}_{\infty}$, there exists $\rho_{1} \in \mathcal{K}_{\infty}$ such that

$$
R^{\circ}(q ; F(q, \xi)) \leq-\rho_{1}(R(q))+\varrho(|\xi|) .
$$

When $\zeta(\tau)>0$, we have that

$$
R(q)=V(x)+\gamma \zeta(\tau) W^{2}(e) .
$$

As above, in view of page 100 in [43], Lemma 1, item (ii) of Assumption 1 and (45),

$$
\begin{aligned}
R^{\circ}(q ; F & (q, \xi))=\langle\nabla V(x), f(x, e, \xi)\rangle+\gamma \dot{\zeta}(\tau) W^{2}(e) \\
& +2 \gamma \zeta(\tau) W(e)\langle\nabla W(e), g(x, e, \xi)\rangle \\
\leq & -\alpha(|x|)-H^{2}(x)-\delta(y)+\gamma^{2} W^{2}(e)+\varrho(|\xi|) \\
& +2 \gamma \zeta(\tau) W(e)(L W(e)+H(x)+\sigma(|\xi|)) \\
& +\gamma W^{2}(e)\left(-2 L \zeta-(1+\kappa) \gamma\left(\zeta^{2}(\tau)+1\right)\right) \\
\leq & -\alpha(|x|)-H^{2}(x)-\delta(y)-\kappa \gamma^{2} W^{2}(e) \\
& +\varrho(|\xi|)-(1+\kappa) \gamma^{2} \zeta^{2}(\tau) W^{2}(e) \\
& +2 \gamma \zeta(\tau) W(e) H(x)+2 \gamma \zeta(\tau) W(e) \sigma(|\xi|) .
\end{aligned}
$$


By using the fact that $2 \gamma \zeta(\tau) W(e) H(x) \leq H^{2}(x)+$ $\gamma^{2} \zeta^{2}(\tau) W^{2}(e), 2 \gamma \zeta(\tau) W(e) \sigma(|\xi|) \leq \frac{1}{\kappa} \sigma^{2}(|\bar{\xi}|)+\kappa \gamma^{2} \times$ $\zeta^{2}(\tau) W^{2}(e)$ and since $\delta(y) \geq 0$, we deduce that $R^{\circ}(q ; F(q, \xi)) \leq-\alpha(|x|)-\kappa \gamma^{2} W^{2}(e)+\chi(|\xi|)$, where $\chi:=\varrho+\frac{1}{\kappa} \sigma^{2}$. By using the same argument as in (47) and since $\zeta(\tau) \leq \theta^{-1}$ for all $\tau \geq 0$ in view of (45), we derive that $R^{\circ}(q ; F(q, \xi)) \leq-\rho_{1}(V(x))-\rho_{2}\left(\gamma \zeta(\tau) W^{2}(e)\right)+$ $\chi(|\xi|)$, where $\rho_{2}: s \mapsto \kappa \gamma \theta s \in \mathcal{K}_{\infty}$. We deduce that there exists $\rho \in \mathcal{K}_{\infty}$ such that

$$
R^{\circ}(q ; F(q, \xi)) \leq-\rho(R(q))+\chi(|\xi|)
$$

where $\rho(s):=\min \left\{\rho_{1}\left(\frac{s}{2}\right), \rho_{2}\left(\frac{s}{2}\right)\right\}$ for $s \geq 0$.

When $\zeta(\tau)=0$, we obtain, in view of (47), (50) and Lemma 1

$$
R^{\circ}(q ; F(q, \xi)) \leq-\rho(R(q))+\chi(|\xi|)
$$

Hence, (51) is satisfied in all cases.

Let $(\phi, \xi)$ be a solution pair to $(8)$, (15) with $\phi:=$ $\left(\phi_{x}, \phi_{e}, \phi_{\tau}\right)$, input $\xi \in \mathcal{L}_{\infty}$ and $\operatorname{dom} \phi=\operatorname{dom} \xi$. In view of (51) and page 99 in [43], for any $\varepsilon \in(0,1), \dot{R}(\phi(t, j)) \leq$ $R^{\circ}(\phi(t, j) ; F(\phi(t, j), \xi(t, j))) \leq-(1-\varepsilon) \rho(R(\phi(t, j)))-$ $\varepsilon \rho(R(\phi(t, j)))+\chi(|\xi(t, j)|)$ for all $j$ and for almost all $t \in I^{j}$ where $I^{j}=\{t:(t, j) \in \operatorname{dom} \phi\}$. As a consequence, using (46) and (51) and by following similar lines as in the end of the proof of Theorem 1 in [35], we deduce that for any $(t, j) \in \operatorname{dom} \phi$

$$
\begin{array}{r}
R(\phi(t, j)) \leq \max \{\bar{\beta}(R(\phi(0,0)), 0.5 t+0.5 T j), \\
\left.\rho^{-1}\left(\frac{1}{\varepsilon} \chi\left(\|\xi\|_{\infty}\right)\right)\right\},
\end{array}
$$

for some $\bar{\beta} \in \mathcal{K} \mathcal{L}$. On the other hand, in view of Assumption 1 and since $W$ is continuous (since it is locally Lipschitz) and positive definite, there exists $\bar{\alpha}_{W} \in \mathcal{K}_{\infty}$ such that $W(e) \leq \bar{\alpha}_{W}(|e|)$ for all $e \in \mathbb{R}^{n_{e}}$ by following similar arguments as in the proof of Lemma 4.3 in [26]. As a result, in view of Assumption 1, (45) and the definition of $R$, it holds that, for all $q \in \mathcal{C} \cup \mathcal{D}$, $R(q) \leq V(x)+\frac{\gamma}{\theta} W^{2}(e) \leq \bar{\alpha}(|x|)+\frac{\gamma}{\theta} \bar{\alpha}_{W}(|e|)=$ : $\bar{\alpha}_{R}(|(x, e)|)$, where $\bar{\alpha}_{R}: s \mapsto \bar{\alpha}(s)+\frac{\gamma}{\theta} \bar{\alpha}_{W}(s) \in$ $\mathcal{K}_{\infty}$. As a result, in view of (52), we deduce that $R(\phi(t, j)) \leq \max \left\{\bar{\beta}\left(\bar{\alpha}_{R}\left(\left|\left(\phi_{x}(0,0), \phi_{e}(0,0)\right)\right|\right), 0.5 t+\right.\right.$ $\left.0.5 T j), \rho^{-1}\left(\frac{1}{\varepsilon} \chi\left(\|\xi\|_{\infty}\right)\right)\right\}$. Consequently, in view of (10), $\left|\phi_{x}(t, j)\right| \leq \underline{\alpha}^{-1}(R(\phi(t, j)))$ and thus (16) holds with $\beta:\left(s_{1}, s_{2}\right) \mapsto \underline{\alpha}^{-1}\left(\bar{\beta}\left(\bar{\alpha}_{R}\left(s_{1}\right), s_{2}\right)\right) \in \mathcal{K} \mathcal{L}$, $\psi(s)=\frac{1}{\varepsilon} \underline{\alpha}^{-1}\left(\rho^{-1}(\chi(s))\right)$ for $s \geq 0$.
Proof of Theorem 3. Let $\zeta_{y}, \zeta_{u}: \mathbb{R}_{\geq 0} \rightarrow \mathbb{R}$ be the solutions to the following differential equations

$$
\begin{array}{ll}
\dot{\zeta}_{y}=-2 L_{y_{1}} \zeta_{y}-\left(1+\kappa_{y}\right) \bar{\lambda}_{y}\left(\zeta_{y}^{2}+1\right), & \zeta_{y}(0)=\theta_{y}^{-1} \\
\dot{\zeta}_{u}=-2 L_{u_{1}} \zeta_{u}-\left(1+\kappa_{u}\right) \bar{\lambda}_{u}\left(\zeta_{u}^{2}+1\right), & \zeta_{u}(0)=\theta_{u}^{-1}
\end{array}
$$

where $\bar{\lambda}_{y}:=\sqrt{\frac{1}{\left(1+\kappa_{y}\right)}\left(\gamma_{y}^{2}+\frac{L_{u_{2}}^{2}}{\kappa_{u}}\right)}, \bar{\lambda}_{u}:=\sqrt{\frac{1}{\left(1+\kappa_{u}\right)}} \times$ $\sqrt{\left(\gamma_{c}^{2}+\frac{L_{y_{2}}^{2}}{\kappa_{y}}\right)}$ for $\kappa_{y}, \kappa_{u}>0$, and $\theta_{y}, \theta_{u} \in(0,1)$. We respectively denote $\widetilde{\mathcal{T}}_{y}$ and $\widetilde{\mathcal{T}}_{c}$ (we dropped the arguments for notational convenience) as the times it takes for $\zeta_{y}, \zeta_{u}$ to decrease from $\theta_{y}^{-1}, \theta_{u}^{-1}$ to $\theta_{y}, \theta_{u}$. By using similar arguments as in (45), we conclude that there exist $\theta_{y}, \theta_{u}>0$ such that $T_{y}<\widetilde{\mathcal{T}}_{y}$ and $T_{u}<\widetilde{\mathcal{T}}_{c}$ and we fix their values.

Let $\mathcal{C}:=\mathcal{C}_{y} \cap \mathcal{C}_{u}$ and $\mathcal{D}:=\mathcal{D}_{y} \cup \mathcal{D}_{u}$. We define for all $q \in \mathcal{C} \cup \mathcal{D}$ (recall that $\left.q=\left(x, e_{y}, e_{c}, \hat{u}, \tau_{y}, \tau_{u}\right)\right), R(q):=$ $V(x)+\max \left\{0, \bar{\lambda}_{y} \zeta_{y}\left(\tau_{y}\right) W_{y}^{2}\left(e_{y}\right)\right\}+\max \left\{0, \bar{\lambda}_{u} \zeta_{u}\left(\tau_{u}\right) W_{c}^{2}\left(e_{c}\right)\right\}$.

By following lines as in (46), we deduce that for all $q \in \mathcal{D}$, $R(G(q)) \leq R(q)$.

Let $q \in \mathcal{C}$. We distinguish five cases in the following.

(i) $\zeta_{y}\left(\tau_{y}\right)<0$ and $\zeta_{u}\left(\tau_{u}\right)<0$. In view of the definition of $R$, we obtain $R(q)=V(x)$ and by using similar arguments as in (47), it holds that $\tau_{y}>T_{y}$ and $\tau_{u}>T_{u}$. Hence, in view of (28) and since $q \in \mathcal{C},\left(\gamma_{y}^{2}+\frac{L_{u_{2}}^{2}}{\kappa_{u}}\right) W_{y}^{2}\left(e_{y}\right) \leq \delta_{y}(y)$ and $\left(\gamma_{c}^{2}+\right.$ $\left.\frac{L_{y_{2}}^{2}}{\kappa_{y}}\right) W_{c}^{2}\left(e_{c}\right) \leq \delta_{c}\left(x_{c}\right)$. Consequently, in view of Assumption $2, R^{\circ}(q ; F(q, \xi))=\langle\nabla V(x), f(x, e)\rangle \leq$ $-|z|^{p}-H_{y}^{2}(x, \xi)-H_{c}^{2}(x, \xi)+\eta^{p}|\xi|^{p} \leq-|z|^{p}+\eta^{p}|\xi|^{p}$.

(ii) $\zeta_{y}\left(\tau_{y}\right)>0$ and $\zeta_{u}\left(\tau_{u}\right)<0$. In this case $R(q)=$ $V(x)+\bar{\lambda}_{y} \zeta_{y}\left(\tau_{y}\right) W_{y}^{2}\left(e_{y}\right)$. In view of Lemma 1 , Assumption 2 and $(53)$, we obtain $R^{\circ}(q ; F(q, \xi))=$ $\langle\nabla V(x), f(x, e, \xi)\rangle+\bar{\lambda}_{y} \dot{\zeta}_{y}\left(\tau_{y}\right) W_{y}^{2}\left(e_{y}\right)+2 \bar{\lambda}_{y} \zeta_{y}\left(\tau_{y}\right) \times$ $W_{y}\left(e_{y}\right)\left\langle\nabla W_{y}\left(e_{y}\right), g_{y}(x, e, \xi)\right\rangle$ which leads to

$$
\begin{aligned}
R^{\circ}(q ; F(q, \xi)) \leq & -|z|^{p}-H_{y}^{2}(x, \xi)-H_{c}^{2}(x, \xi) \\
& -\delta_{y}(y)-\delta_{c}\left(x_{c}\right)+\gamma_{y}^{2} W_{y}^{2}\left(e_{y}\right) \\
& +\gamma_{c}^{2} W_{c}^{2}\left(e_{c}\right)+\eta^{p}|\xi|^{p} \\
& -\left(1+\kappa_{y}\right) \bar{\lambda}_{y}^{2} \zeta_{y}^{2}\left(\tau_{y}\right) W_{y}^{2}\left(e_{y}\right) \\
& -\left(1+\kappa_{y}\right) \bar{\lambda}_{y}^{2} W_{y}^{2}\left(e_{y}\right) \\
& +2 \bar{\lambda}_{y} \zeta_{y}\left(\tau_{y}\right) W_{y}\left(e_{y}\right) L_{y_{2}} W_{c}\left(e_{c}\right) \\
& +2 \bar{\lambda}_{y} \zeta_{y}\left(\tau_{y}\right) W_{y}\left(e_{y}\right) H_{y}(x, \xi) .
\end{aligned}
$$


Using the fact that $2 \bar{\lambda}_{y} \zeta_{y}\left(\tau_{y}\right) W_{y} L_{y_{2}} W_{c}\left(e_{c}\right) \leq$ $\kappa_{y} \bar{\lambda}_{y}^{2} \zeta_{y}^{2}\left(\tau_{y}\right) W_{y}^{2}\left(e_{y}\right)+\frac{L_{y_{2}}^{2}}{\kappa_{y}} W_{c}^{2}\left(e_{c}\right)$ and $2 \bar{\lambda}_{y} \zeta_{y}\left(\tau_{y}\right) \times$ $W_{y}\left(e_{y}\right) H_{y}(x, \xi) \leq \bar{\lambda}_{y}^{2} \zeta_{y}^{2}\left(\tau_{y}\right) W_{y}^{2}\left(e_{y}\right)+H_{y}^{2}(x, \xi)$ and $\left(\gamma_{c}^{2}+\frac{L_{y_{2}}^{2}}{\kappa_{y}}\right) W_{c}^{2}\left(e_{c}\right) \leq \delta_{c}\left(x_{c}\right)$ since $q \in \mathcal{C}$ and $\zeta_{u}\left(\tau_{u}\right)<0$, we have that (recall that $\left(1+\kappa_{y}\right) \bar{\lambda}_{y}^{2}=$ $\left.\gamma_{y}^{2}+\frac{L_{u_{2}}^{2}}{\kappa_{u}}\right), R^{\circ}(q ; F(q, \xi)) \leq-|z|^{p}+\eta^{p}|\xi|^{p}$.

(iii) $\zeta_{y}\left(\tau_{y}\right)<0$ and $\zeta_{u}\left(\tau_{u}\right)>0$. By following the same lines as in the previous case, we deduce that $R^{\circ}(q ; F(q, \xi)) \leq-|z|^{p}+\eta^{p}|\xi|^{p}$.

(iv) $\zeta_{y}\left(\tau_{y}\right)>0$ and $\zeta_{u}\left(\tau_{u}\right)>0$. In view of the definition of $R, R(q)=V(x)+\bar{\lambda}_{y} \zeta_{y}\left(\tau_{y}\right) W_{y}^{2}\left(e_{y}\right)+$ $\bar{\lambda}_{u} \zeta_{u}\left(\tau_{u}\right) W_{c}^{2}\left(e_{c}\right)$. By following similar lines as in case (ii), we obtain $R^{\circ}(q ; F(q, \xi)) \leq-|z|^{p}+\eta^{p}|\xi|^{p}$.

(v) $\zeta_{y}\left(\tau_{y}\right)=0$ or $\zeta_{u}\left(\tau_{u}\right)=0$. In view of cases (i)-(iv) and Lemma 1, we obtain $R^{\circ}(q ; F(q, \xi)) \leq-|z|^{p}+$ $\eta^{p}|\xi|^{p}$.

As a result, it holds that, for all $q \in \mathcal{C}_{y} \cap \mathcal{C}_{u}$

$$
R^{\circ}(q ; F(q, \xi)) \leq-|z|^{p}+\eta^{p}|\xi|^{p} .
$$

Let $(\phi, \xi)$ be a solution pair to (19), (28) with $\phi:=\left(\phi_{x}, \phi_{e_{y}}, \phi_{e_{c}}, \phi_{\hat{u}}, \phi_{\tau_{y}}, \phi_{\tau_{u}}\right)$, input $\xi \in \mathcal{L}_{p}$ and $\operatorname{dom} \phi=\operatorname{dom} \xi$. In view of (55) and page 99 in [43], it holds that $\dot{R}(\phi(t, j)) \leq R^{\circ}(\phi(t, j) ; F(\phi(t, j), \xi(t, j))) \leq$ $-|z(t, j)|^{p}+\eta^{p}|\xi(t, j)|^{p}$ for all $j$ and for almost all $t \in I^{j}$ where $I^{j}=\{t:(t, j) \in \operatorname{dom} \phi\}$. Finally, by following similar lines as in the end of the proof of Theorem 1 and Theorem IV.7 in [23], we conclude that $\|z(t, j)\|_{p} \leq \bar{\alpha}_{R}(\phi(0,0))^{1 / p}+\eta\|\xi(t, j)\|_{p}$, where $\bar{\alpha}_{R}: s \mapsto \bar{\alpha}(s)+\frac{\bar{\lambda}_{y}}{\theta_{y}} \bar{\alpha}_{W_{y}}(s)+\frac{\bar{\lambda}_{u}}{\theta_{u}} \bar{\alpha}_{W_{c}}(s) \in \mathcal{K}_{\infty}$. Thus, the conclusions of Theorem 3 hold.

Proof of Proposition 1: Let $W_{y}\left(e_{y}\right)=\lambda_{y}\left|e_{y}\right|$ and $W_{c}\left(e_{c}\right)=\lambda_{u}\left|e_{c}\right|$. Then we have, for all $x \in \mathbb{R}^{n_{x}}$ and almost all $\left(e_{y}, e_{c}\right) \in \mathbb{R}^{n_{y}+n_{c}},\left\langle\nabla W_{y}\left(e_{y}\right), \mathcal{A}_{2} x+\mathcal{B}_{2} e_{y}+\right.$ $\left.\mathcal{M}_{2} e_{c}+\mathcal{E}_{2} \xi\right\rangle \leq \lambda_{y}\left|\mathcal{A}_{2} x+\mathcal{E}_{2} \xi\right|+\lambda_{y}\left|\mathcal{B}_{2}\right|\left|e_{y}\right|+\lambda_{y}\left|\mathcal{M}_{2}\right|\left|e_{c}\right|$ and $\left\langle\nabla W_{c}\left(e_{c}\right), \mathcal{A}_{3} x+\mathcal{B}_{3} e_{y}+\mathcal{E}_{3} \xi\right\rangle \leq \lambda_{u}\left|\mathcal{A}_{3} x+\mathcal{E}_{3} \xi\right|+$ $\lambda_{u}\left|\mathcal{B}_{3}\right|\left|e_{y}\right|$. Hence, condition (24) holds with $H_{y}(x, \xi)=$ $\lambda_{y}\left|\mathcal{A}_{2} x+\mathcal{E}_{2} \xi\right|, H_{c}(x, \xi)=\lambda_{u}\left|\mathcal{A}_{3} x+\mathcal{E}_{3} \xi\right|, L_{y_{1}}=$ $\left|\mathcal{B}_{2}\right|, L_{y_{2}}=\frac{\lambda_{y}}{\lambda_{u}}\left|\mathcal{M}_{2}\right|, L_{u_{1}}=0, L_{u_{2}}=\frac{\lambda_{u}}{\lambda_{y}}\left|\mathcal{B}_{3}\right|$.

Let $V(x)=x^{T} P x$, for $x \in \mathbb{R}^{n_{x}}$. Condition (22) is satisfied with $\underline{\alpha}(s)=\lambda_{\min }(P) s^{2}$ and $\bar{\alpha}(s)=\lambda_{\max }(P) s^{2}$ for $s \geq 0$. In view of (32), it holds that, for all $\left(e_{y}, e_{c}\right) \in \mathbb{R}^{n_{e}}$ and all $x \in \mathbb{R}^{n_{x}},\left\langle\nabla V(x), \mathcal{A}_{1} x+\mathcal{B}_{1} e_{y}+\mathcal{M}_{1} e_{c}+\mathcal{E}_{1} \xi\right\rangle=$ $x^{T}\left(\mathcal{A}_{1}^{T} P+P \mathcal{A}_{1}\right) x+2 x^{T} P \mathcal{B}_{1} e_{y}+2 x^{T} P \mathcal{M}_{1} e_{c}+2 x^{T} P \mathcal{E}_{1} \xi$. By post- and pre-multiplying LMI (34) respectively by the state vector $\left(x, e_{y}, e_{c}, \xi\right)$ and its transpose and by rearranging the terms we obtain $\left\langle\nabla V(x), \mathcal{A}_{1} x+\mathcal{B}_{1} e_{y}+\right.$
$\left.\mathcal{M}_{1} e_{c}+\mathcal{E}_{1} \xi\right\rangle \leq-|z|^{2}-H_{y}^{2}(x, \xi)-H_{c}^{2}(x, \xi)-\varepsilon_{y}|y|^{2}-$ $\varepsilon_{c}\left|x_{c}\right|^{2}+\frac{\mu_{y}}{\lambda_{y}^{2}} W_{y}^{2}\left(e_{y}\right)+\frac{\mu_{u}}{\lambda_{u}^{2}} W_{c}^{2}\left(e_{c}\right)+\vartheta|\xi|^{2}$. As a result, condition (23) is verified with $\delta_{y}(y)=\varepsilon_{y}|y|^{2}, \delta_{c}\left(x_{c}\right)=$ $\varepsilon_{c}\left|x_{c}\right|^{2}, \gamma_{y}=\frac{\sqrt{\mu_{y}}}{\lambda_{y}}, \gamma_{c}=\frac{\sqrt{\mu_{u}}}{\lambda_{u}}$, and $\eta=\sqrt{\vartheta}$.

\section{References}

[1] M. Abdelrahim, R. Postoyan, and J. Daafouz. Eventtriggered control of nonlinear singularly perturbed systems based only on the slow dynamics. Automatica, 52:15-22, 2015.

[2] M. Abdelrahim, R. Postoyan, J. Daafouz, and D. Nešić. Stabilization of nonlinear systems using event-triggered output feedback laws. IEEE Transactions on Automatic Control, available on-line.

[3] M. Abdelrahim, R. Postoyan, J. Daafouz, and D. Nešić. Co-design of output feedback laws and event-triggering conditions for linear systems. In Proceedings of the 53rd IEEE Conference on Decision and Control, Los Angeles, U.S.A., pages 3560-3565, 2014.

[4] M. Abdelrahim, R. Postoyan, J. Daafouz, and D. Nešić. Stabilization of nonlinear systems using event-triggered output feedback laws. In Proceedings of the 21th International Symposium on Mathematics Theory of Networks and Systems, Groningen, The Netherlands, pages 274-281, 2014

[5] M. Abdelrahim, R. Postoyan, J. Daafouz, and D. Nešić. Event-triggered dynamic feedback controllers for nonlinear systems with asynchronous transmissions. In Proceedings of the 54th IEEE Conference on Decision and Control, Osaka, Japan, pages 5494-5499, 2015.

[6] M. Abdelrahim, R. Postoyan, J. Daafouz, and D. Nešić. Input-to-state stabilization of nonlinear systems using eventtriggered output feedback controllers. In Proceedings of the 14th European Control Conference, Linz, Austria, pages 2185-2190, 2015.

[7] A. Angeli, E.D. Sontag, and Y. Wang. Input-to-state stability with respect to inputs and their derivatives. International Journal of Robust and Nonlinear Control, 13(11):1035-1056, 2003.

[8] A. Anta and P. Tabuada. To sample or not to sample: Selftriggered control for nonlinear systems. IEEE Transactions on Automatic Control, 55(9):2030-2042, 2010.

[9] P. Antsaklis and J. Baillieul. Special issue on technology of networked control systems. In Proceedings of the IEEE, 95(1):5-8, 2007.

[10] D. Antunes, J. Hespanha, and C. Silvestre. Stability of networked control systems with asynchronous renewal links: An impulsive systems approach. Automatica, 49(2):402-413, 2013.

[11] K.E. Årzén. A simple event-based PID controller. In Proceedings of the $14^{\text {th }}$ IFAC World Congress, Beijing, China, 18:423-428, 1999.

[12] K.J. Åström and B.M. Bernhardsson. Comparison of periodic and event based sampling for first order stochastic systems. In Proceedings of the 14th IFAC World Congress, Beijing, China, pages 301-306, 1999.

[13] J. Baillieul and P.J. Antsaklis. Control and communication challenges in networked real-time systems. Proceedings of the IEEE, 95(1):9-28, 2007. 
[14] D.P. Borgers and W.P.M.H. Heemels. Event-separation properties of event-triggered control systems. IEEE Transactions on Automatic Control, 59(10):2644-2656, 2014.

[15] C. Cai and A.R. Teel. Characterizations of input-to-state stability for hybrid systems. Systems \& Control Letters, 58(1):47-53, 2009.

[16] D. Carnevale, A.R. Teel, and D. Nešić. A Lyapunov proof of an improved maximum allowable transfer interval for networked control systems. IEEE Transactions on Automatic Control, 52(5):892-897, 2007.

[17] V.S. Dolk, D.P. Borgers, and W.P.M.H. Heemels. Outputbased and decentralized dynamic event-triggered control with guaranteed $\mathcal{L}_{p}$-gain performance and zeno-freeness. IEEE Transactions on Automatic Control, to appear.

[18] M.C.F. Donkers and W.P.M.H. Heemels. Outputbased event-triggered control with guaranteed $\mathcal{L}_{\infty}$-gain and improved and decentralised event-triggering. IEEE Transactions on Automatic Control, 57(6):1362-1376, 2012.

[19] F. Forni, S. Galeani, D. Nešić, and L. Zaccarian. Eventtriggered transmission for linear control over communication channels. Automatica, 50(2):490-498, 2014.

[20] R. Goebel, R.G. Sanfelice, and A.R. Teel. Hybrid Dynamical Systems: Modeling, Stability, and Robustness. Princeton University Press, 2012.

[21] W.P.M.H. Heemels, D.P. Borgers, N. van de Wouw, D. Nešić, and A.R. Teel. Stability analysis of nonlinear networked control systems with asynchronous communication: A smallgain approach. In Proceedings of the 52nd IEEE Conference on Decision and Control, Firenze, Italy, pages 4631-4637, 2013.

[22] W.P.M.H. Heemels, K.H. Johansson, and P. Tabuada. An introduction to event-triggered and self-triggered control. In Proceedings of the 51st IEEE Conference on Decision and Control, Maui, U.S.A., pages 3270-3285, 2012.

[23] W.P.M.H. Heemels, A.R. Teel, N. van de Wouw, and D. Nešić. Networked control systems with communication constraints: Tradeoffs between transmission intervals, delays and performance. IEEE Transactions on Automatic Control, 55(8):1781-1796, 2010.

[24] S.H.J. Heijmans, R. Postoyan, N. Noroozi, D. Nešić, and W.P.M.H. Heemels. Stability analysis of networked control systems with direct-feedthrough terms: Part II - the linear case. Submitted to the 55th IEEE Conference on Decision and Control.

[25] K.H. Johansson, J. Lygeros, S. Sastry, and M. Egerstedt. Simulation of zeno hybrid automata. In Proceedings of the 38th IEEE Conference on Decision and Control, Phoenix, U.S.A., pages 3538-3543, 1999.

[26] H.K. Khalil. Nonlinear Systems. Prentice Hall, 3rd edition, 2002.

[27] D. Lehmann, G.A. Kiener, and K.H. Johansson. Eventtriggered PI control: Saturating actuators and anti-windup compensation. In Proceedings of the 51st Conference on Decision and Control, Maui, U.S.A., pages 6566-6571, 2012.

[28] D. Lehmann and J. Lunze. Event-based output-feedback control. In Proceedings of the 19th Mediterranean Conference on Control and Automation, Corfu, Greece, pages 982-987, 2011.

[29] D. Liberzon, D. Nešić, and A.R. Teel. Lyapunov-based smallgain theorems for hybrid systems. IEEE Transactions on Automatic Control, 59(6):1395-1410, 2014.

[30] J. Lunze and D. Lehmann. A state-feedback approach to event-based control. Automatica, 46:211-215, 2010.
[31] M. Mazo Jr., A. Anta, and P. Tabuada. An ISS self-triggered implementation of linear controllers. Automatica, 46:13101314,2010

[32] M. Mazo Jr. and P. Tabuada. Decentralized event-triggered control over wireless sensor/actuator networks. IEEE Transactions on Automatic Control, 56(10):2456-2461, 2011.

[33] D. Nešić and D.S. Laila. A note on input-to-state stabilization for nonlinear sampled-data systems. IEEE Transactions on Automatic Control, 47(7):1153-1158, 2002.

[34] D. Nešić and A.R. Teel. Input-output stability properties of networked control systems. IEEE Transactions on Automatic Control, 49(10):1650-1667, 2004.

[35] D. Nešić, A.R. Teel, and D. Carnevale. Explicit computation of the sampling period in emulation of controllers for nonlinear sampled-data systems. IEEE Transactions on Automatic Control, 54(3):619-624, 2009.

[36] N. Noroozi, R. Postoyan, D. Nešić, S.H.J. Heijmans, and W.P.M.H. Heemels. Stability analysis of networked control systems with direct-feedthrough terms: Part I - the nonlinear case. Submitted to the 55th IEEE Conference on Decision and Control.

[37] C. De Persis and P. Tesi. Input-to-state stabilizing control under denial-of-service. IEEE Transactions on Automatic Control, 60(11):2930-2944, 2015.

[38] R. Postoyan, P. Tabuada, D. Nešić, and A. Anta. A framework for the event-triggered stabilization of nonlinear systems. IEEE Transactions on Automatic Control, 60(4):982-996, 2015.

[39] R.G. Sanfelice, J.J.B. Biemond, N. van de Wouw, and W.P.M.H. Heemels. An embedding approach for the design of state-feedback tracking controllers for references with jumps. International Journal of Robust and Nonlinear Control, 24(11):1585-1608, 2014.

[40] C.W. Scherer and S. Weiland. Lecture notes, Linear matrix inequalities in control. The Netherlands: Dutch Institute for Systems and Control (DISC), 1999.

[41] P. Tabuada. Event-triggered real-time scheduling of stabilizing control tasks. IEEE Transactions on Automatic Control, 52(9):1680-1685, 2007.

[42] P. Tallapragada and N. Chopra. Event-triggered dynamic output feedback control for LTI systems. In Proceedings of the 51st IEEE Conference on Decision and Control, Maui, U.S.A., pages 6597-6602, 2012.

[43] A.R. Teel and L. Praly. On assigning the derivative of a disturbance attenuation control Lyapunov function. Mathematics of Control, Signal and Systems, 13(2):95-124, 2000.

[44] M. Velasco, J. Fuertes, and P. Marti. The self triggered task model for real-time control systems. In Proceedings of the 24th IEEE Real-Time Systems Symposium (RTSS WIP 2003), pages 67-70, 2003.

[45] X. Wang and M. Lemmon. Self-triggered feedback control systems with finite-gain $\mathcal{L}_{2}$ stability. IEEE Transactions on Automatic Control, 45:452-467, 2009.

[46] X. Wang, Y. Sun, and N. Hovakimyan. Asynchronous task execution in networked control systems using decentralized event-triggering. Systems \& Control Letters, 61(9):936-944, 2012. 


\section{University Library}

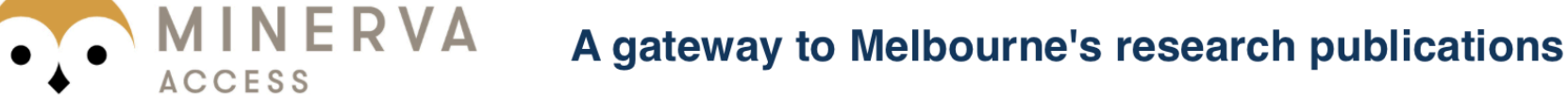

Minerva Access is the Institutional Repository of The University of Melbourne

Author/s:

Abdelrahim, M;Postoyan, R;Daafouz, J;Nesic, D

Title:

Robust event-triggered output feedback controllers for nonlinear systems

Date:

2017-01-01

Citation:

Abdelrahim, M., Postoyan, R., Daafouz, J. \& Nesic, D. (2017). Robust event-triggered output feedback controllers for nonlinear systems. Automatica, 75, pp.96-108. https:// doi.org/10.1016/j.automatica.2016.09.044.

Persistent Link:

http://hdl.handle.net/11343/251860 